\title{
A new lepto-hadronic model applied to the first simultaneous multiwavelength data set for Cygnus X-1
}

\author{
D. Kantzas ${ }^{\oplus},{ }^{1,2 \star}$ S. Markoff ${ }^{\oplus}, 1,2$ T. Beuchert,${ }^{3,1}$ M. Lucchini ${ }^{\oplus},{ }^{1}$ A. Chhotray, ${ }^{1}$ C. Ceccobello, ${ }^{4}$ \\ A. J. Tetarenko ${ }^{\oplus}, 5$ J. C. A. Miller-Jones ${ }^{\oplus},{ }^{6}$ M. Bremer, ${ }^{7}$ J. A. Garcia, ${ }^{8,9}$ V. Grinberg, ${ }^{10}$ P. Uttley ${ }^{1}$ \\ and J. Wilms ${ }^{9}$ \\ ${ }^{1}$ Anton Pannekoek Institute for Astronomy (API), University of Amsterdam, Science Park 904, NL-1098 XH Amsterdam, the Netherlands \\ ${ }^{2}$ GRavitational AstroParticle Physics Amsterdam (GRAPPA), University of Amsterdam, Science Park 904, NL-1098 XH Amsterdam, the Netherlands \\ ${ }^{3}$ European Southern Observatory, Karl-Schwarzschild-Straße 2, D-85748 Garching bei München, Germany \\ ${ }^{4}$ Department of Space, Earth and Environment, Chalmers University of Technology, Onsala Space Observatory, SE-439 92 Onsala, Sweden \\ ${ }^{5}$ East Asian Observatory, 660 N. A'ohōkū Place, University Park, Hilo, Hawaii 96720, USA \\ ${ }^{6}$ International Centre for Radio Astronomy Research - Curtin University, GPO Box U1987, Perth, WA 6845, Australia \\ ${ }^{7}$ Institut de Radio Astronomie Millimétrique (IRAM), 300 rue de la Piscine, F-38406 Saint Martin d'Hères, France \\ ${ }^{8}$ Cahill Center for Astronomy and Astrophysics, Caltech, 1200 East California Boulevard, Pasadena, CA 91125, the USA \\ ${ }^{9}$ Dr. Karl Remeis-Observatory and Erlangen Centre for Astroparticle Physics, Sternwartstr. 7, D-96049 Bamberg, Germany \\ ${ }^{10}$ Institute for Astronomy und Astrophysics, University of Tübingen, Sand 1, D-72076 Tübingen, Germany
}

Accepted 2020 October 20. Received 2020 October 16; in original form 2020 March 3

\begin{abstract}
Cygnus $\mathrm{X}-1$ is the first Galactic source confirmed to host an accreting black hole. It has been detected across the entire electromagnetic spectrum from radio to $\mathrm{GeV}$ gamma-rays. The source's radio through mid-infrared radiation is thought to originate from the relativistic jets. The observed high degree of linear polarization in the MeV X-rays suggests that the relativistic jets dominate in this regime as well, whereas a hot accretion flow dominates the soft X-ray band. The origin of the $\mathrm{GeV}$ non-thermal emission is still debated, with both leptonic and hadronic scenarios deemed to be viable. In this work, we present results from a new semi-analytical, multizone jet model applied to the broad-band spectral energy distribution of Cygnus $\mathrm{X}-1$ for both leptonic and hadronic scenarios. We try to break this degeneracy by fitting the first-ever high-quality, simultaneous multiwavelength data set obtained from the CHOCBOX campaign (Cygnus X-1 Hard state Observations of a Complete Binary Orbit in X-rays). Our model parametrizes dynamical properties, such as the jet velocity profile, the magnetic field, and the energy density. Moreover, the model combines these dynamical properties with a self-consistent radiative transfer calculation including secondary cascades, both of leptonic and hadronic origin. We conclude that sensitive $\mathrm{TeV}$ gamma-ray telescopes like Cherenkov Telescope Array (CTA) will definitively answer the question of whether hadronic processes occur inside the relativistic jets of Cygnus X-1.
\end{abstract}

Key words: acceleration of particles - radiation mechanisms: non-thermal-X-rays: individual: Cyg X-1.

\section{INTRODUCTION}

Throughout the Universe, a significant fraction of accreting black holes are known to launch relativistic and collimated jets. Fundamental properties, such as the extent and power of these jets, scale essentially with the mass of the central black hole. While supermassive black holes (SMBHs) with $M_{\mathrm{BH}} \sim 10^{6}-10^{9} \mathrm{M}_{\odot}$ located at the centre of active galactic nuclei (AGNs) are able to power jets up to Mpc scales (e.g. Waggett, Warner \& Baldwin 1977), Galactic black holes $\left(M_{\mathrm{BH}} \sim\right.$ tens of $\left.\mathrm{M}_{\odot}\right)$ hosted by X-ray binaries (XRBs) typically launch jets that remain collimated up to sub-pc scales (e.g. Mirabel \& Rodriguez 1994; Hjellming \& Rupen 1995; Mioduszewski et al. 2001; Gallo et al. 2005; Fender et al. 2006; Rushton et al. 2017; Russell et al. 2019).

^E-mail: d.kantzas@uva.nl
AGN jets carry enough power to accelerate particles up to ultrahigh energies of $10^{19} \mathrm{eV}$ and above (Aharonian 2000), which we detect as cosmic rays (CRs) on Earth. The exact acceleration mechanism is not known, but is likely related to diffusive shock acceleration (Axford 1969; Blandford \& Ostriker 1978; Ellison, Jones \& Reynolds 1990; Rieger, Bosch-Ramon \& Duffy 2007), magnetic re-connection (Spruit, Daigne \& Drenkhahn 2001; Giannios 2010; Sironi, Petropoulou \& Giannios 2015), or shearing and instabilities at boundary layers between different velocities (Rieger \& Duffy 2004; Liu, Rieger \& Aharonian 2017).

The CR spectrum detected on Earth covers more than ten orders of magnitude in particle energy, from $10^{9}$ to $\sim 10^{21} \mathrm{eV}$. Two wellknown characteristic spectral features of that spectrum are the socalled knee at $10^{15} \mathrm{eV}$ and the 'ankle' at $10^{18} \mathrm{eV}$ (Kulikov \& Khristiansen 1959; Bird et al. 1993, respectively). As shown by Hillas (1984), the maximum energy of the accelerated particles at a given magnetic field is limited by the size of the source due 
to confinement arguments. Accordingly, CRs above the ankle are likely of extragalactic origin, whereas CRs below the knee are of Galactic origin. AGN jets are considered the most likely source of extragalactic CRs (e.g. Hillas 1984; Gaisser, Engel \& Resconi 2016; Eichmann et al. 2018, and references therein). Supernovae and supernova remnants have been considered the dominant source of Galactic CRs for decades although questioned quite recently due to lack of $\geq 100 \mathrm{TeV}$ observations (Aharonian, Yang \& de Oña Wilhelmi 2019). Hence, new candidate sources are needed.

Large AGN jets and small-scale XRB jets are (self-)similar in many regards. For example, they display similar non-thermal emission processes, suggesting that both classes are capable of accelerating particles to high energies regardless of their physical scales (e.g. Markoff, Falcke \& Fender 2001; Bosch-Ramon, Romero \& Paredes 2006; Zdziarski, Lubiński \& Sikora 2012). Recent observations of hydrogen and helium emission lines from the jets of the accreting compact object SS 433 (Fabrika 2004), as well as the iron emission lines from the stellar-mass black hole candidate 4U 1630-47 (Díaz Trigo et al. 2013), provide indirect evidence of hadronic content their jets. It is still not clear whether XRB jets can efficiently accelerate hadrons to high energy, but if so, they could also be potential Galactic CRs sources (see e.g. Heinz \& Sunyaev 2002; Fender, Maccarone \& van Kesteren 2005; Cooper et al. 2020).

The most striking evidence for particle acceleration inside Galactic jets comes from the non-thermal $\mathrm{GeV}$ radiation detected by the XRBs Cygnus X-1 (Cyg X-1) and Cygnus X-3 (Tavani et al. 2009; Malyshev, Zdziarski \& Chernyakova 2013; Bodaghee et al. 2013; Zanin et al. 2016). The jet-origin of the $\mathrm{GeV}$ emission is further favoured by the orbital modulation predicted, e.g. by Böttcher \& Dermer (2005). Zdziarski et al. (2017) in fact detected an MeV-GeV modulation that likely originates from synchrotron self-Compton upscattering by particles accelerated in the compact black hole-jet system of Cyg X-1 orbiting its companion star.

The exact nature of the non-thermal radiation is still unclear, with both leptonic and hadronic processes deemed to be viable. In the former case, a leptonic population is responsible for the overall electromagnetic spectrum from radio to gamma-rays (e.g. BoschRamon et al. 2006). In the latter case, the hadronic population reaches relativistic speeds as well and contributes equally, or even dominates, in the high-energy regime of the spectrum. According to the Hillas criterion, particles can attain high-enough energy only if a strong magnetic field confines them in the acceleration region and provides enough power for particle acceleration. The power carried by accelerated protons has been claimed to exceed the Eddington luminosity in several cases making the hadronic model controversial (Zdziarski \& Böttcher 2015). The hadronic channel, however, is the only possible way to explain the observed high and ultra-high energy CRs, as well as neutrinos through particle cascades (e.g. Mannheim \& Schlickeiser 1994; Aharonian 2002).

The modelling of either of these radiative processes requires knowledge of the geometrical structure of the emitting region. Observations show jets that remain collimated up to large distances, following cylindrical or conical structures (e.g. Lister et al. 2013; Hada et al. 2016). However, for simplicity, spectral models often consider localized and spherical single-zone accelerating regions because they provide a good first-order approximation (e.g. Tavecchio, Maraschi \& Ghisellini 1998; Mastichiadis \& Kirk 2002; Marscher et al. 2008). In order to correctly factor in the observed jet geometry, we need to describe an accelerating and expanding outflow, and properly connect its physical properties with those of the accretion flow. Such inhomogeneous multizone jet models are able to self- consistently produce both the characteristic flat-to-inverted radio spectra observed in many compact jet systems, and the upscattered high-energy continuum (Blandford \& Königl 1979; Hjellming \& Johnston 1988).

Multiple groups have considered such multizone models in the past. For instance, Falcke \& Biermann (1995) derived a simple model for the dynamical properties of a hydrodynamically driven, selfcollimating jet, assumed to be powered by the accretion flow. This model was further developed with jet-intrinsic particle distributions and more detailed radiative calculations, and extended to XRBs by Markoff et al. (2001) and Markoff, Nowak \& Wilms (2005). The semi-analytical nature of this model has the great advantage that one can directly fit its physical parameters to data. Numerical simulations of the detailed magnetohydrodynamics of the jet flow, combined with radiative transfer calculations, would be very computational expensive and time-consuming for such a task.

In this work, we adopt the multizone leptonic model of Markoff et al. (2005) in its most recent version (Maitra et al. 2009; Crumley et al. 2017; Lucchini et al. 2019; 2020) and we further develop it by including hadronic interactions. This is the first hadronic multizone jet model for Galactic sources that additionally includes further improvements to the already implemented leptonic ones, such as pair cascades (Coppi \& Blandford 1990; Böttcher \& Schlickeiser 1997).

An ideal source to test our newly developed model is one of the brightest and well-studied black hole high-mass XRB, Cyg X-1, and its persistent jets (Stirling et al. 2001; Rushton et al. 2012). Along with the model, we present a new data set obtained by the CHOCBOX campaign (Cyg X-1 Hard state Observations of a Complete Binary Orbit in X-rays: Uttley 2017). This campaign performed simultaneous observations with the satellite observatories XMM-Newton, NUSTAR, and INTEGRAL, which, together with the ground-based interferometers (NOEMA, VLA, and VLBA), provide the first multiwavelength data set of that kind for Cyg X-1.

We also include the most recent $\mathrm{X}$-ray polarization information for Cyg X-1. Linear polarization has been reported in the energy band below $200 \mathrm{keV}$ but the polarization fraction is strongly energydependent and does not exceed 10 per cent (Chauvin et al. 2018a, b). In contrast, the hard X-ray emission in the $0.4-2 \mathrm{MeV}$ band is linearly polarized at a level of $\sim 70$ per cent (Laurent et al. 2011; Jourdain et al. 2012; Rodriguez et al. 2015). Such a high polarization fraction can only be explained as synchrotron emission from an ordered magnetic field, and places strong constraints on the modelling. In this work, we assume that the synchrotron radiation originates in the compact jets of Cyg X-1.

For this work, we adopt the updated distance and black hole mass for Cyg X-1 of $2.22 \mathrm{kpc}$ and $21.4 \mathrm{M}_{\odot}$, respectively (Miller-Jones et al. 2020). The distance is in good agreement with the Gaia DR2 distance of $2.38_{-0.17}^{+0.20}$ (Brown et al. 2018; Gandhi et al. 2019), which is about 30 per cent more distant than previously thought (Reid et al. 2011). The mass of the black hole was historically estimated to be between 14.8 $\mathrm{M}_{\odot}$ (Orosz et al. 2011) and $16 \mathrm{M}_{\odot}$ (Ziółkowski 2014; Mastroserio, Ingram \& van der Klis 2019), significantly lower than the updated value. The impact of the updated value of the mass of the black hole can be significant, making the revision of modelling the source necessary. The jet inclination angle is $27.5^{\circ}$. The companion is a $\sim 41 \mathrm{M}_{\odot}$ star (Miller-Jones et al. 2020), which is about twice as massive as the foregoing estimate by Orosz et al. (2011). The spectral type of the companion star is O9.7 Iab (Bolton 1972). The binary separation is estimated to be $\sim 3.7 \times 10^{12} \mathrm{~cm}$ (Miller et al. 2005) and the system orbital period is around $5.6 \mathrm{~d}$ (Webster \& Murdin 1972). 
Table 1. The observational multiwavelength data used in this work.

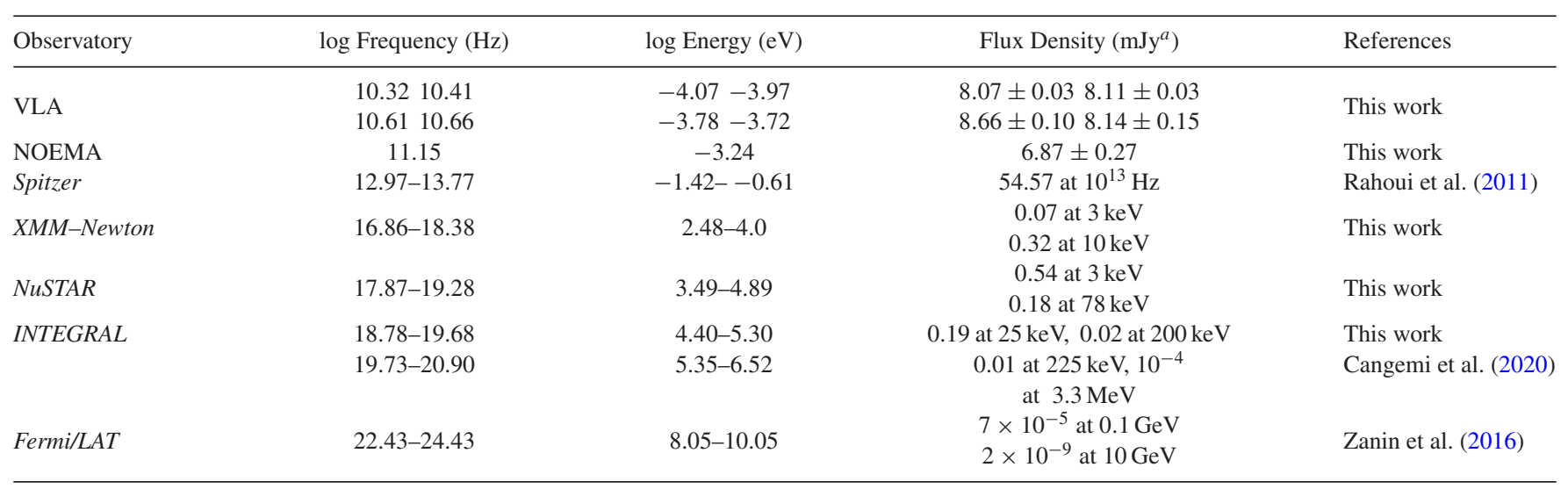

Note. ${ }^{a} \mathrm{mJy}=10^{-26} \mathrm{erg} \mathrm{cm}^{-2} \mathrm{~s}^{-1} \mathrm{~Hz}^{-1}$.

This paper is organized as follows. We discuss the new observational data set of Cyg X-1 in Section 2 and our new lepto-hadronic model in Section 3. In Section 4, we present the results of our modelling. Finally, we outline in Section 5 the significance of the results and summarize our work in Section 6.

\section{OBSERVATIONS AND DATA EXTRACTION}

The bulk of the data we use to constrain the physical parameters of our model resulted from the CHOCBOX campaign (Uttley 2017). In particular, we select data within the time interval 2016 May 31 05:15:01.5 - 07:07:04.5 UTC, which provides simultaneous coverage by NOEMA, XMM-Newton, NUSTAR, and INTEGRAL.

In addition, we consider some supplemental, non-simultaneous, long-term averaged archival data. We use the mid-infrared data (Rahoui et al. 2011) to constrain physical properties of the donor star. We take into account a long-term 15 -yr average $\mathrm{MeV}$ spectrum by INTEGRAL (Cangemi et al. 2020) as well as the publicly available $\mathrm{GeV}$ gamma-ray spectrum from the Fermi/LAT collaboration (Zanin et al. 2016). The low flux and challenging detection techniques require averaging the data over longer timescales. Cangemi et al. (2020) are the first to average over all existing INTEGRAL data of Cyg X-1 in its hard state. The gamma-ray spectrum we use here comprises data averaged over $7.5 \mathrm{yr}$, only during the hard state of Cyg X-1. Averaging thus provides the best-possible constraints to the $\mathrm{MeV}$ and $\mathrm{GeV}$ emission at the moment. While modelling, we do take into account the systematics arising from integrating over flux variations. We list all the data we use in this work in Table 1.

\subsection{Very Large Array (VLA)}

We observed Cyg X-1 with the Karl G. Jansky Very Large Array (VLA) on 2016 May 31, from 04:29-08:28 UT, under project code VLA/15B-236. The VLA observed in two sub-arrays of 14 and 13 antennas spread approximately evenly over each of the three arms of the array, which was in its moderately-extended B configuration. The first subarray observed primarily in the $Q$ band, with two $1024-\mathrm{MHz}$ basebands centred at 40.5 and $46.0 \mathrm{GHz}$, and the second observed primarily in the $K$ band, with the two $1024-\mathrm{MHz}$ basebands centred at 20.9 and $25.8 \mathrm{GHz}$. Each subarray observed a single 2-min scan at a lower frequency (two 1024-MHz basebands centred at 5.25 and $7.45 \mathrm{GHz}$, and a single $1024 \mathrm{MHz}$ baseband centred at $1.5 \mathrm{GHz}$, respectively) to characterize the broadband spectral behaviour. We used 3C 286 as the bandpass and delay calibrator, and to set the flux density scale, and we derived the complex gain solutions using the nearby extragalactic source $\mathrm{J} 2015+3710$.

We processed the data using the Common Astronomy Software Application (CASA; McMullin et al. 2007). The data were initially calibrated using the VLA CASA Calibration Pipeline (v4.5.3), and after some additional flagging to excise radio frequency interference, we imaged the target data using CASA version 4.5.2. The low elevation at the beginning of the run caused significant phase decorrelation and an increased system temperature. Although we were able to selfcalibrate the data in phase down to a solution time-scale of $2 \mathrm{~min}$, the flux densities were still found to be biased low. We therefore restricted our images to the final 90 min of the run. Cygnus X-1 was significantly detected in all images, which were made with Briggs weighting, with a robust parameter of 1 .

\subsection{NOrthern Extended Millimetre Array (NOEMA)}

The NOEMA observations of Cyg X-1 (project code: W15BQ, PI: Tetarenko) took place on 2016 May 31 (05:15:01-07:52:53.0 UT, MJD 57539.2188-57539.3284), in the $2 \mathrm{~mm}$ (tuning frequency of $140 \mathrm{GHz}$ ) band. These observations were made with the WideX correlator, to yield 1 base-band, with a total bandwidth of $3.6 \mathrm{GHz}$ per polarization. The array was in the 6ant-Special configuration (N02W12E04N11E10N07), with 6 antennas, spending $1.9 \mathrm{hr}$ on source during our observations. We used $\mathrm{J} 2013+370$ as a phase calibrator, 3C 454.3 as a bandpass calibrator, and MWC349 as a flux calibrator. We performed phase only self-calibration on the data, with a solution interval of $45 \mathrm{~s}$. The weather significantly degraded after 07:07 UT at NOEMA; therefore, we do not include data after that time in our analysis. As CASA is unable to handle NOEMA data in its original format, flagging and calibration of the data were first performed in GILDAS ${ }^{1}$ using standard procedures, then the data were exported to $\mathrm{CASA}^{2}$ for imaging (with natural weighting to maximize sensitivity). The flux density of the source was measured by fitting a point source in the image plane (using the imfit task).

\footnotetext{
${ }^{1}$ http://www.iram.fr/IRAMFR/GILDAS

${ }^{2}$ To convert a NOEMA data set for use in CASA, we followed the procedures outlined at https://www.iram.fr/IRAMFR/ARC/ documents/filler/casa-gilda s.pdf.
} 


\subsection{XMM-Newton}

We consider the XMM-Newton observation ID 0745250501, which observed Cyg X-1 in timing mode using its EPIC-pn camera (Strüder et al. 2001) for a total of about $145 \mathrm{ks}$. First, we create calibrated and filtered event lists using the SAS V.16.1.0, which we further correct for X-ray loading and flag soft flare events. We consider only counts strictly simultaneous to the NOEMA observation time period resulting in a net exposure time of $3.5 \mathrm{ks}$. We use the filtered event lists to extract $0.3-10 \mathrm{keV}$ spectra according to standard procedures.

\subsection{NUSTAR}

NuSTAR (Harrison et al. 2013) measures photons up to $\sim 80 \mathrm{keV}$ by focusing hard X-rays on two focal-plane modules FPM A and FPM B. We extract data from within $3-78 \mathrm{keV}$ with the standard NUSTAR Data Analysis Software NuSTARDAS-v .1.8.0 as part of HEASOFT-V $\cdot 6.22$. 1. Due to the high flux of Cyg X-1, we extract source counts from within a relatively large region of 150 arcsec radius on both chips FPM A and FPM B, and background counts from a region of 100 arcsec located off-source but close enough not prevent bias due to the spatial background dependence (Wik et al. 2014). To make sure to have simultaneous coverage with the observational time window of NOEMA, we define appropriate goodtime intervals for the observation ID 30002150004, which results in a net exposure time of $1.9 \mathrm{ks}$ each for FPM A and FPM B.

\subsection{INTEGRAL}

We extract the INTEGRAL Soft Gamma-Ray Imager (ISGRI; Lebrun et al. 2003) data with the Off-line Scientific Analysis (OSA) software v10.2 to match the simultaneous time interval as much as possible, resulting in the use of three science windows, 168500020010,168500030010 , and 168500040010 and $6.5 \mathrm{ks}$ effective exposure time.

The state-resolved scientific products (images, light curves, and spectra) of the coded-mask instrument ISGRI were obtained with standard procedures. We extract spectra and images of Cyg X-1 on a single-science-window ( $\mathrm{scw}$ ) basis. For each scw, we construct a sky model including the brightest sources active in the field at the time of observation as found from the analysis of the full CHOCBOX INTEGRALexposure, i.e. Cyg X-1, Cyg X-3, Cyg A, GRO J2058+42, KS 1947+300, and SAX J2103.5+4545.

\section{MODEL DETAILS}

\subsection{Dynamical quantities}

We describe the multizone jet model based on Markoff et al. (2005) and its extensions referenced above. In this section, we summarize the major properties of the model and focus on our new extension of including the effect of hadronic particle acceleration and secondary production.

A fully self-consistent jet model should solve the force balance equations along the streamlines and perpendicular to them. This calculation would yield the radial profile and the acceleration profile describing a given jet configuration starting from a set of initial conditions. For simplicity, we assume a fixed shape for the jet radial profile, based on observational evidence in AGN, which together with the longitudinal velocity profile then determines the profiles along the jet of the number density, and global magnetic field strength. Specifically, the cross-sectional radius $R$ at any height $z$ along the jet is given by

$R(z)=R_{0}+\left(z-z_{0}\right) \frac{\Gamma_{0} \beta_{0}}{\Gamma_{j} \beta_{j}}$,

where $R_{0}$ is the radius of the jet base, $z_{0}$ is the height of the jet base above the black hole, $\beta_{0, j}$ and $\beta_{j}$ are the bulk velocity of the plasma at the jet base and at height $z$, respectively, and $\Gamma$ is the corresponding Lorentz factor.

The solution of the Euler equation (Crumley et al. 2017)

$$
\begin{gathered}
\left\{\Gamma_{j} \beta_{j} \frac{\Gamma_{\mathrm{ad}}+\xi}{\Gamma_{\mathrm{ad}}-1}-\Gamma_{\mathrm{ad}} \Gamma_{j} \beta_{j}-\frac{\Gamma_{\mathrm{ad}}}{\Gamma_{j} \beta_{j}}+\frac{2\left(z-z_{0}\right) \Gamma_{0} \beta_{0} /\left(\Gamma_{j} \beta_{j}\right)}{R_{0} \Gamma_{j} \beta_{j}+\Gamma_{0} \beta_{0}\left(z-z_{0}\right)}\right\} \\
\times \frac{\partial \Gamma_{j} \beta_{j}}{\partial z}=\frac{2 \Gamma_{0} \beta_{0}}{R_{0} \Gamma_{j} \beta_{j}+\Gamma_{0} \beta_{0}\left(z-z_{0}\right)}
\end{gathered}
$$

gives the velocity profile along the jet $\Gamma_{j}(z)$. In the above equation, $\Gamma_{\mathrm{ad}}$ is the adiabatic index of the flow $(5 / 3$ for a non-relativistic and $4 / 3$ for a relativistic flow),

$\xi=\left(\frac{\Gamma_{j} \beta_{j}}{\Gamma_{0} \beta_{0}}\right)^{\Gamma_{\mathrm{ad}}-1} ; \Gamma_{0} \beta_{0}=\sqrt{\frac{\Gamma_{\mathrm{ad}}\left(\Gamma_{\mathrm{ad}}-1\right)}{1+2 \Gamma_{\mathrm{ad}}-\Gamma_{\mathrm{ad}}^{2}}}$.

Conservation of the particle number density results in

$n(z)=n_{0}\left(\frac{\Gamma_{j} \beta_{j}}{\Gamma_{0} \beta_{0}}\right)^{-1}\left(\frac{R}{R_{0}}\right)^{-2}$,

where $n_{0}$ is the differential number density at the jet base in $\mathrm{cm}^{-3} \mathrm{erg}^{-1}$. For a quasi-isothermal jet, which seems to be necessary to explain the flat/inverted spectrum, the internal energy density is given by (see Crumley et al. 2017)

$U_{j}(z)=n_{0} m_{\mathrm{p}} c^{2}\left(\frac{\Gamma_{\mathrm{j}} \beta_{\mathrm{j}}}{\Gamma_{0} \beta_{0}}\right)^{-\Gamma_{\mathrm{ad}}}\left(\frac{R}{R_{0}}\right)^{-2}$,

where $m_{\mathrm{p}} c^{2}$ is the rest-frame energy of the protons that carry most of the kinetic energy. By assuming a fixed plasma beta parameter $\beta=$ $U_{\mathrm{e}} / U_{\mathrm{B}}$, where $U_{\mathrm{e}}$ is the internal energy density of the electrons, and $U_{\mathrm{B}}$ the magnetic energy density, we can determine the profile of the magnetic field along the jet to be

$B(z)=\sqrt{\frac{8 \pi U_{\mathrm{e}}(z)}{\beta}}$,

where the energy density of the magnetic field is $U_{\mathrm{B}}=B^{2} / 8 \pi$. For simplicity, we do not distinguish between toroidal and poloidal components but we assume that the field is tangled with a characteristic strength.

In addition to the jets, which include a thermal-dominated, coronalike region at their base, we incorporate a simple description for an additional thermal compact corona located around the black hole. We assume that a hot electron plasma of temperature $T_{\text {cor }}$ is covering a radius $R_{\text {cor }}$ and has an optical depth $\tau_{\text {cor }}$. These hot electrons inverse Compton upscatter the blackbody photons emitted by the accretion disc, while the thermal population in the jet base can upscatter both disc photons as well as synchrotron photons.

\subsection{Particle distributions}

Thermal electrons ${ }^{3}$ are assumed to be directly injected into the jet base from the accreting inflow with a thermal Maxwell-Jüttner distribution, which reduces to the standard Maxwellian form in the

\footnotetext{
${ }^{3}$ We do not distinguish between electrons and positrons. The results in this work do not depend on the charge of the lepton.
} 
non-relativistic case. Protons can be found in the jet base as well but they are entirely cold, and only carry the kinetic energy of the jet. The initial number density of the protons carried by the jet is defined as

$n_{0}=\frac{L_{\text {jet }}}{4 \beta_{0, s} \Gamma_{0, s} c \mathrm{~m}_{\mathrm{p}} \mathrm{c}^{2} \pi \mathrm{R}_{0}^{2}}$,

where half of the injected power $L_{\text {jet }}$ goes into cold protons, while the other half is shared by the magnetic field and leptons, thus the factor of $1 / 4$. We assume equal number density of electrons and protons. Further, $\beta_{0, s} \Gamma_{0, s} c$ is the sound speed of a relativistic fluid with adiabatic index $4 / 3$. The total injected power $L_{\text {jet }}$ is a free parameter of the model and is assumed to be proportional to the accretion energy $\dot{M} c^{2}$.

Once the particles propagate out some distance $z_{\text {diss }}$ along the jet, a fitted parameter, we assume that a fixed fraction (10 per cent) of both leptons and hadrons are accelerated into a power law with index $p$ from this point onwards. We do not invoke any particular acceleration mechanism nor distinguish between acceleration or re-acceleration. We thus allow the power-law index $p$ to be a free parameter in our model. Moreover, we assume constant particle acceleration beyond the particle acceleration region $z_{\text {diss }}$. Another free parameter is the acceleration efficiency $f_{\text {sc }}$ (see e.g. Jokipii 1987; Aharonian 2004). Given this efficiency, the maximum energy achieved by the particles is calculated self-consistently along the jet by considering the main physical processes that limit the further acceleration of particles. The dominant cooling mechanisms are synchrotron radiation and inverse Compton scattering (ICS) for leptons, and escape from the source for hadrons. Adiabatic cooling is not relevant because the jets are actively collimated.

In order to calculate, the particle distributions along the jets, we solve the continuity equation, which in energy phase space can be written in the general form:

$$
\begin{array}{r}
\frac{\partial N_{i}\left(E_{i}, t, z\right)}{\partial t}+\frac{\partial\left(\Gamma_{j} v_{j} N\left(E_{i}, t, z\right)\right)}{\partial z} \\
+\frac{\partial\left(b\left(E_{i}, t, z\right) N_{i}\left(E_{i}, t, z\right)\right)}{\partial E_{i}}-\frac{N_{i}\left(E_{i}, t, z\right)}{\tau_{\text {esc }}\left(E_{i}, t, z\right)}=Q\left(E_{i}, t, z\right) .
\end{array}
$$

The above equation describes the temporal evolution of the number density of the particle population $i$, i.e. electrons or protons. Since we assume a steady-state source, we neglect the first term on the lefthand side, making every quantity time-independent. We also neglect the effects of spallation and diffusion.

The second term on the left-hand side describes the propagation of particles along the jet. The third term expresses the radiative cooling of the particles, i.e. synchrotron radiation and ICS for leptons, as well as inelastic collisions for hadrons. The particles may escape the source within the time-scale $\tau_{\text {esc }}\left(E_{i}, t, z\right)$, which in our treatment is only energy dependent. Finally, the right-hand side describes the injection term, which is the sum of a Maxwell-Jüttner thermal distribution at low energies and a non-thermal power law with an exponential cut-off at the self-consistently derived maximum energy. The non-thermal power law is included only starting at the dissipation region $z_{\text {diss }}$ where particle acceleration initiates.

Losses will dominate over acceleration above some particular energy $E_{\max }$ which can be self-consistently calculated - here for the leptonic case - by setting

$\tau_{\text {acc }}^{-1}\left(E_{e, \text { max }}\right)=\tau_{\text {syn }}^{-1}\left(E_{e, \text { max }}\right)+\tau_{\text {ICS }}^{-1}\left(E_{e, \max }\right)+\tau_{\text {esc }}^{-1}\left(E_{e, \max }\right)$,

with the time-scales for acceleration, synchrotron cooling, ICS cooling in the Thomson regime, and the escape of leptons, i.e.
(i) $\tau_{\mathrm{acc}}=\frac{4 E_{e}}{3 f_{\mathrm{sc}} \mathrm{ecB}}$,
(ii) $\tau_{\mathrm{syn}}=\frac{6 \pi \mathrm{m}_{\mathrm{e}}^{2} \mathrm{c}^{3}}{\sigma_{\mathrm{T}} B^{2} E_{e} \beta_{e}^{2}}$,
(iii) $\tau_{\mathrm{ICS}}=\tau_{\mathrm{syn}} \frac{U_{B}}{u_{\mathrm{rad}}}$,
(iv) $\tau_{\mathrm{esc}}=\frac{R}{\beta_{e} \mathrm{c}}$,

respectively. Here, e is the electron charge, $B$ is the magnetic field of the jet at height $z$ with radius $R, m_{\mathrm{e}}$ is the rest mass of the electron, $c$ is the speed of light, $\sigma_{\mathrm{T}}$ is the Thomson cross-section, $\beta_{e}$ is the speed of the particle in units of c, $U_{\mathrm{B}}=B^{2} / 8 \pi$ the energy density of the magnetic field, $u_{\mathrm{rad}}$ the energy density of the radiation field upscattered by the electrons.

Following the same approach, we calculate the maximum energy of protons in case of hadronic acceleration by setting

$$
\begin{array}{r}
\tau_{\mathrm{acc}}^{-1}\left(E_{p, \max }\right)=\tau_{\mathrm{syn}}^{-1}\left(E_{p, \max }\right)+\tau_{\mathrm{pp}}^{-1}\left(E_{p, \max }\right)+ \\
\tau_{\mathrm{p} \gamma}^{-1}\left(E_{p, \max }\right)+\tau_{\mathrm{esc}}^{-1}\left(E_{p, \max }\right),
\end{array}
$$

with the time-scales for acceleration, synchrotron cooling, protonproton collisions, proton-photon collisions, and the escape of protons, i.e.

$$
\begin{aligned}
& \text { (i) } \tau_{\mathrm{acc}}=\frac{4 E_{p}}{3 f_{\mathrm{sc}} \mathrm{ecB}}, \\
& \text { (ii) } \tau_{\mathrm{syn}}=\frac{6 \pi \mathrm{m}_{\mathrm{p}}^{2} \mathrm{c}^{3}}{\sigma_{\mathrm{T}} B^{2} E_{p} \beta_{p}^{2}} \times\left(\frac{\mathrm{m}_{\mathrm{p}}}{\mathrm{m}_{\mathrm{e}}}\right)^{2}, \\
& \text { (iii) } \tau_{\mathrm{pp}}=\left(K_{\mathrm{pp}} \sigma_{\mathrm{pp}} n_{\mathrm{th}} c\right)^{-1}, \\
& \text { (iv) } \tau_{p \gamma}=\left(K_{\mathrm{p} \gamma} \sigma_{\mathrm{p} \gamma} n_{\gamma} c\right)^{-1}, \\
& \text { (v) } \tau_{\mathrm{esc}}=\frac{R}{\beta_{p} c} .
\end{aligned}
$$

Here, $K_{\mathrm{pp}}$ corresponds to the multiplicity (average number of secondary particles), $\sigma_{\mathrm{pp}}$ to the cross-section of this interaction, and $n_{\text {th }}$ to the number density of the target particles (see Section 3.3.2). For proton-photon interactions between the accelerated protons and a photon field with number density $n_{\gamma}$, we consider the multiplicity $K_{\mathrm{p} \gamma}$ (Mannheim \& Schlickeiser 1994). One can see that the protonsynchrotron time-scale is approximately $\left(m_{\mathrm{p}} / m_{\mathrm{e}}\right)^{3}$ times longer than the electron one.

The injection term becomes a power law with an exponential cutoff beyond the particle acceleration region $z_{\text {diss }}$, i.e.

$Q\left(E_{i}\right)=Q_{0} E_{i}^{-p} \times \exp \left(-E_{i} / E_{i, \max }\right)$,

where $Q_{0}$ is a normalization factor and $p>0$ is allowed to vary between 1.5 and 2.5 , consistent with standard particle acceleration mechanisms. The power-law index is assumed to be equal for electrons and protons, which implies a common acceleration mechanism for both populations. Equation (11) is the less computationally expensive form of the output of Particle-In-Cell (PIC) simulations where the thermal particle distribution leads to a self-consistent formation of a power law of accelerated particles in time (e.g. Sironi \& Spitkovsky 2009; Crumley et al. 2019; and references therein). We include further distributions of secondary pairs from hadronic processes and photon-photon annihilation (see below) into this injection term $Q$. 


\subsection{Radiative processes}

\subsubsection{Leptonic processes}

Electrons throughout the jet lose energy due to synchrotron and IC radiation. Before the particle acceleration region, even thermal electrons emit synchrotron radiation due to the relatively strong magnetic field. Beyond the particle acceleration region, the nonthermal leptonic process that dominates is the synchrotron radiation. For electron ICS, we include photon fields from synchrotron radiation (synchrotron-self Compton - SSC), the disc around the black hole, and the companion star. We take into account the geometry of the companion star because, for high-mass XRBs like Cyg X-1, the size of the star is comparable to the size of the jet, especially for regions close to the compact object where the majority of the high-energy radiation is likely to originate. In particular, we calculate the photon field of the companion star as seen in the jet frame accounting for the Doppler boosting (each jet segment travels at a different Lorentz factor). All expressions for synchrotron radiation and ICS are taken from Blumenthal \& Gould (1970) and Rybicki \& Lightman (2008).

Furthermore, we include the full treatment of photon-photon annihilation and electromagnetic cascades (Coppi \& Blandford 1990; Böttcher \& Schlickeiser 1997). Depending on the number density of produced pairs, additional interactions between electrons and positrons can cause pair-annihilation leading to the production of gamma-rays. This process can occur until the lepton energy budget becomes insufficient for further photon production. The photon fields we take into account are the same as for ICS. Finally, we add the produced pairs to the leptonic population, which are then cooled as described above.

\subsubsection{Hadronic processes}

In the case where protons and/or ions are accelerated to relativistic energies in the jet, they can inelastically collide with thermal protons and photons inside the jet flow and produce secondary particles (Mannheim \& Schlickeiser 1994). In the extension of our model, we therefore implement both proton-proton and proton-photon interactions. We use the full semi-analytical treatment of Kelner, Aharonian \& Bugayov (2006) and Kelner \& Aharonian (2008) based on Monte Carlo simulations (see below for more details).

3.3.2.1 Proton-proton interactions Collisions of non-thermal protons with thermal jet protons and stellar-wind protons (proton-proton collisions, pp, henceforth) lead to the production of gamma-rays, secondary electrons, and neutrinos. The interactions responsible for the production of these particles can be described as

$\mathrm{p}+\mathrm{p} \rightarrow \mathrm{p}+\mathrm{p}+\alpha \pi^{0}+\beta\left(\pi^{+}+\pi^{-}\right)$,

where $\alpha$ and $\beta$ are the collision energy-dependent multiplicity of the related products (see e.g. Romero et al. 2017). The charged pions decay as

$\pi^{+} \rightarrow \mu^{+}+v_{\mu}, \quad \mu^{+} \rightarrow \mathrm{e}^{+}+v_{\mathrm{e}}+\bar{v}_{\mu}$,

$\pi^{-} \rightarrow \mu^{-}+\bar{v}_{\mu}, \quad \mu^{-} \rightarrow \mathrm{e}^{-}+\bar{v}_{\mathrm{e}}+v_{\mu}$,

and the neutral pions decay into two gamma-rays, i.e.

$\pi^{0} \rightarrow \gamma+\gamma$

In order for these interactions to occur, the energy of the accelerated proton has to exceed the threshold of $E_{\mathrm{th}} \simeq 1.22 \mathrm{GeV}$ (Mannheim \& Schlickeiser 1994).
The lifetime of the produced mesons is well measured by laboratory experiments and short compared to the dynamical time-scales of the jet. We can therefore assume instant decays. Consequently, the charged products do not radiatively lose energy as they would in extreme environments of either very strong magnetic fields or very high energies (e.g. Mücke et al. 2003). The above statement can be parametrized as follows (e.g. Böttcher et al. 2013)

$\mathrm{B} \gamma_{\mathrm{p}} \ll\left\{\begin{array}{l}7.8 \times 10^{11} \mathrm{G} \text { for pions } \\ 5.6 \times 10^{10} \mathrm{G} \text { for muons, }\end{array}\right.$

where $B$ is the strength of the magnetic field in the jet rest frame and $\gamma_{\mathrm{p}}$ the Lorentz factor of the proton. Given that the highest value of the magnetic field is in the jet base $\left(10^{7} \mathrm{G}\right)$ and that hadronic interactions do not occur yet because particle acceleration occurs later, one can see that the above inequality is always satisfied.

In order to produce the distributions of stable products, we follow the semi-analytical approximation of Kelner et al. (2006). In particular, the differential number density of the gamma-rays is given by the expression:

$$
\begin{aligned}
\frac{\mathrm{d} n_{\gamma}\left(z, E_{\gamma}\right)}{\mathrm{d} E_{\gamma}}= & c n_{\mathrm{targ}} \int_{0}^{1} \sigma_{\mathrm{pp}}\left(\frac{E_{\gamma}}{x}\right) n_{\mathrm{p}}\left(z, \frac{E_{\gamma}}{x}\right) \\
& \times F_{\gamma}\left(x, \frac{E_{\gamma}}{x}\right) \frac{\mathrm{d} x}{x},
\end{aligned}
$$

where $E_{\gamma}$ is the energy of the gamma-ray, $n_{\text {targ }}$ is the number density of the thermal target protons, $\sigma_{\mathrm{pp}}$ is the cross-section for pp collisions, $n_{\mathrm{p}}$ is the number density of the non-thermal protons, $x=E_{\gamma} / E_{\mathrm{p}}$ is the normalized photon energy with respect to initial proton energy, and $\mathrm{F}_{\gamma}\left(\mathrm{x}, \mathrm{E}_{\gamma} / \mathrm{x}\right)$ is the spectrum of gamma-rays.

The cross-section for pp interactions can be given by the semianalytical expression

$$
\begin{array}{r}
\sigma_{\mathrm{pp}}\left(T_{p}\right)=\left[30.7-0.96 \log \left(\frac{T_{p}}{T_{\mathrm{thr}}}\right)+0.18 \log ^{2}\left(\frac{T_{p}}{T_{\mathrm{thr}}}\right)\right] \\
\times\left[1-\left(\frac{T_{\mathrm{thr}}}{T_{p}}\right)^{1.9}\right]^{3} \mathrm{mb},
\end{array}
$$

where $T_{p}$ is the proton kinetic energy in the laboratory frame and $T_{\text {thr }}=2 m_{\pi}+m_{\pi}^{2} / 2 m_{p} \simeq 0.2797 \mathrm{GeV}$ the threshold kinetic energy for this interaction to take place (Kafexhiu et al. 2014). Kelner et al. (2006) provide semi-analytical calculations for the gammaray spectrum as well as the other secondary particles.

For this work, the target protons are the cold protons of the jet and protons emitted by the heavy companion star in the form of a homogeneous stellar wind. In particular, the companion star of Cyg $\mathrm{X}-1$ is a blue supergiant that loses $\sim 10^{-6} \mathrm{M}_{\odot} \mathrm{yr}^{-1}$ in the form of stellar wind (Gies et al. 2008). We use the following expression to calculate the proton number density emitted by the companion

$n_{\text {wind }}(z)=\frac{\dot{M}_{\star}}{4 \pi\left(\alpha_{\star}^{2}+z^{2}\right) v_{\text {wind }} m_{\mathrm{p}}} \times\left[1-\frac{R_{\star}}{\sqrt{\alpha_{\star}^{2}+z^{2}}}\right]^{-\beta_{\text {wind }}}$

(Grinberg et al. 2015), where $\dot{M}_{\star}=4 \pi \rho(r) v(r)$ is the mass-loss rate based on the radially dependent mass density profile $\rho(z), v_{\text {wind }}$ is the terminal velocity of the wind on the jet wall, $\alpha_{\star}^{2}$ is the distance of the massive star from the black hole, $R_{\star}$ is the radius of the massive star, $z$ is the distance from the central black hole along the jet axis, and $\beta_{\text {wind }}$ is a free parameter used to improve the velocity profile of the wind found to be 1.6 (see e.g. Grinberg et al. 2015). From geometrical, filling-factor considerations, we assume that only 10 per cent of the wind protons take part in the pp process (see e.g. Pepe, Vila \& Romero 2015). Therefore, the total target number 
Table 2. The fixed parameters of our models.

\begin{tabular}{|c|c|c|}
\hline Parameter & Value & Description \\
\hline$M_{\mathrm{BH}}\left(\mathrm{M}_{\odot}\right)$ & 21.4 & Mass of the black hole ${ }^{a}$ \\
\hline$\theta_{\text {incl }}$ & $27.5^{\circ}$ & Viewing anglea $^{a}$ \\
\hline$D(\mathrm{kpc})$ & $\begin{array}{c}2.22 \\
0.6\end{array}$ & $\begin{array}{l}\text { Distance of the source }{ }^{a} \\
\text { Number column density }\end{array}$ \\
\hline \multicolumn{3}{|l|}{$N_{H}\left(10^{22} \mathrm{~cm}^{-2}\right)$} \\
\hline$h=z_{0} / R_{0}$ & 2 & Initial jet height to radius ratio \\
\hline$z_{\max }\left(r_{\mathrm{g}}\right)$ & $10^{8}$ & Maximum jet height ${ }^{b}$ \\
\hline$T_{\star}(K)$ & $3.08 \times 10^{4}$ & Temperature of the companion $\operatorname{star}^{a}$ \\
\hline$L_{\star}\left(\mathrm{erg} \mathrm{s}^{-1}\right)$ & $1.57 \times 10^{39}$ & Luminosity of the companion $\operatorname{star}^{a}$ \\
\hline$a_{\star}(\mathrm{cm})$ & $3.7 \times 10^{12}$ & Orbital separation distance ${ }^{\mathrm{a}}$ \\
\hline$\dot{M}_{\star}\left(\mathrm{M}_{\odot} \mathrm{yr}^{-1}\right)$ & $2.6 \times 10^{-6}$ & Mass-loss rate of the companion $\operatorname{star}^{c}$ \\
\hline$v_{\text {wind }}\left(\mathrm{cm} \mathrm{s}^{-1}\right)$ & $2.4 \times 10^{8}$ & Velocity of the stellar wind ${ }^{c}$ \\
\hline
\end{tabular}

Note. ${ }^{a}$ Miller-Jones et al. (2020), ${ }^{b}$ Tetarenko et al. (2019). ${ }^{c}$ similar to Grinberg et al. (2015)

density $\left(\right.$ in $\mathrm{cm}^{-3}$ ) is given by

$n_{\text {targ }}(z)=0.1 n_{\text {wind }}(z)+n_{\mathrm{p}, \text { cold }}(z)$.

3.3.2.2 Proton-photon interactions In addition to the pp interaction, inelastic collisions between non-thermal protons and photons occur in the jet ( $\mathrm{p} \gamma$ henceforth). For this process, we take into account the same photons fields as described above for leptonic ICS.

Depending on the centre-of-mass energy of the inelastic collision, we consider two processes: photopair and photomeson interactions. The photopair interaction is a $\mathrm{p} \gamma$ collision resulting in the production of an electron-positron pair

$\mathrm{p}+\gamma \rightarrow \mathrm{p}+\mathrm{e}^{+}+\mathrm{e}^{-}$,

also called the Bethe-Heitler process. Alternatively, a $\mathrm{p} \gamma$ collision can result in the production of mesons, similarly to the pp interaction discussed above. The photomeson process can be written as

$\mathrm{p}+\gamma \rightarrow \mathrm{p}+\mathrm{p}+\alpha \pi^{0}+\beta\left(\pi^{+}+\pi^{-}\right)$.

The energy thresholds for photopair and photomeson processes to occur are as follows:

$E_{p, \text { thres }}=4.8 \times 10^{14} / \epsilon_{\mathrm{eV}} \mathrm{eV}$ for photopair,

$E_{p \text {,thres }}=7.1 \times 10^{16} / \epsilon_{\mathrm{eV}} \mathrm{eV}$ for photomeson,

where $\epsilon_{\mathrm{eV}}$ is the energy of the target photon in $\mathrm{eV}$. The photopair process has a lower energy threshold to occur. However, if the energy threshold for the photomeson process is met, then the energy loss of the proton is more significant compared to the photopair process, making the photomeson process dominant (Mannheim \& Schlickeiser 1994).

Semi-analytical expressions for the distributions of stable secondary particles are provided by Kelner \& Aharonian (2008). Secondary particles produced in the above processes can further interact within the jet before escaping. In this paper, we do not add the secondary leptons to the primary leptonic population, but rather calculate their radiative processes and their relative contribution to the electromagnetic spectrum separately, for comparison.

\subsection{Corona model}

Along with the jet, we include an additional component in the form of a simple spherical corona surrounding the accretion disc. As discussed in Section 4.2, this is necessary in order to match the $\mathrm{X}$-ray emission of the source.
We assume that the electrons in the corona are thermal with a temperature $T_{\text {cor }}$ and that the entire corona is described by an optical depth $\tau_{\text {cor }}$ and a radius $R_{\text {cor }}$. We define the number density of the injected electrons as $n_{\mathrm{e}, \text { cor }}=\tau_{\text {cor }} / \sigma_{\mathrm{T}} R_{\text {cor }}$, where $\sigma_{\mathrm{T}}$ is the Thomson cross-section. For the emission related to the corona, we only consider the disc photons as the source of seed photons for ICS, and we calculate the radiation energy density of the seed photons at the centre of the system. This means that the coronal radius $R_{\text {cor }}$ effectively acts as a normalization constant, rather than representing the exact physical radius of the $\mathrm{X}$-ray emitting region.

\section{RESULTS}

We perform simultaneous spectral fits of all data presented in Section 2 using the Interactive Spectral Interpretation System ( IS IS; Houck \& Denicola 2000). We explore the parameter space using a Markov Chain Monte Carlo (MCMC) method and its implementation via the emcee algorithm. In particular, we initiate 20 walkers per free parameter and perform $\sim 10^{4}$ loops. The chains require a significant number of loops before they successfully converge, so we exclude the 50 per cent of the initial loops. We use the rest of the loops to derive the uncertainties of each free parameter (shown in Table 3). The fixed parameters including those of the donor star as assumed by Grinberg et al. (2015) are given in Table 2. The free parameters we allow to vary during the fitting are shown in Table 3 . These are the injected power to the jet base $L_{\mathrm{jet}}$, the radius of the jet base $R_{0}$, the location where the particle acceleration initiates $z_{\text {diss }}$, the plasma beta parameter $\beta$, the parameters for the disc, namely the innermost radius $R_{\mathrm{in} \text {, disc }}$ and the mass accretion rate in Eddington units $\left(\dot{m}=\dot{M} \mathrm{c}^{2} / L_{\mathrm{Edd}}\right)$, and the parameters of the corona, namely the temperature $T_{\text {cor }}$, the normalization radius $R_{\text {cor }}$ and the optical depth $\tau_{\text {cor }}$.

We present here the results of the best fits of our models. We choose one lepto-hadronic and one purely leptonic model to reproduce the $\mathrm{MeV} \mathrm{X}$-rays as jet synchrotron radiation, so as to explain the high degree of linear polarization (Laurent et al. 2011; Jourdain et al. 2012; Rodriguez et al. 2015; Cangemi et al. 2020). We achieve this by assuming that the non-thermal electrons accelerate in a hard power law. We find that an index of $p=1.7$ provides sufficient results. We show two more models for comparison. One purely leptonic and one lepto-hadronic, with softer power laws of $p=2.2$. With such an assumption we fail to reproduce the $\mathrm{MeV}$ polarization as we show below.

\subsection{Plasma quantities}

The four different models presented here lead to different jet dynamical quantities, as we show in Table 3 . The jet base radius varies between 2 and $27 r_{\mathrm{g}}$ and the region where the energy dissipates into particle acceleration varies between 15 and $125 r_{\mathrm{g}}$. The two models with a hard injected particle distribution require a small value of plasma $\beta$ compared to the softer models.

The best-fitting values for the injected power $L_{\text {jet }}$ for the models with the hard power law $(p=1.7)$ are comparable. Based on the jet-base radius $R_{0}$ and the plasma $\beta$, we calculate the strength of the magnetic field along the jet. For all our models, we find relatively high magnetic field strengths at the jet base on the order of $10^{6} \mathrm{G}$.

In Fig. 1, we plot the energy density of various quantities along the jet axis for models the two models with $p=1.7$. In particular, our fits are driven towards particle-dominated jets with the energy density of the protons dominating along the jet. Moreover, the energy 
Table 3. The free parameters of the four models discussed in this paper that differ in the power-law index $p$ of the accelerated particles. Before the double line, we show the fitted parameters and their uncertainties. Below, we show the evaluated quantities of the magnetic field, the total luminosity of the accelerated proton/electron population, and the maximum energy of the protons/electrons at the particle acceleration region.

\begin{tabular}{|c|c|c|c|c|c|}
\hline \multirow{2}{*}{$\begin{array}{l}\text { Parameter } \\
p\end{array}$} & \multicolumn{2}{|c|}{ Lepto-hadronic models } & \multicolumn{2}{|c|}{ Leptonic models } & \multirow{2}{*}{$\begin{array}{l}\text { Description } \\
\text { Particle power-law index }\end{array}$} \\
\hline & 1.7 & 2.2 & 1.7 & 2.2 & \\
\hline$L_{\text {jet }}\left(\begin{array}{c}\left(10^{-4} L_{\text {Edd }}\right) \\
\left(\mathrm{erg} \mathrm{s}^{-1}\right)\end{array}\right.$ & $9_{-5}^{+26}$ & $105_{-6}^{+360}$ & $7.1_{-2.5}^{+3.4}$ & $2.0_{-1.9}^{+2.1}$ & Jet base injected power \\
\hline$R_{0}\left(r_{\mathrm{g}}\right)$ & $27_{-25}^{+29}$ & $2^{+21}$ & $3.1_{-1.1}^{+12.5}$ & $3.1_{-1.1}^{+4.1}$ & Jet base radius \\
\hline$z_{\text {diss }}\left(r_{\mathrm{g}}\right)$ & $81_{-15}^{+114}$ & $15_{-4}^{+496}$ & $64_{-14}^{+57}$ & $125_{-45}^{+475}$ & Particle acceleration region \\
\hline$\dot{m}\left(10^{-3}\right)$ & $2.5_{-2.4}^{+15.9}$ & $1.22_{-1.20}^{+1.25}$ & $1.4_{-1.3}^{+1.5}$ & $1.1_{-1.0}^{+1.2}$ & Mass accretion rate \\
\hline$R_{\text {in, disc }}\left(r_{\mathrm{g}}\right)$ & $19_{-17}^{+11}$ & $3.1_{-1.1}^{+3.3}$ & $6.3_{-4.3}^{+6.5}$ & $3.1_{-1.1}^{+3.3}$ & Disc innermost radius \\
\hline$T_{\text {cor }}(\mathrm{keV})$ & $90_{-10}^{+69}$ & $89_{-9}^{+16}$ & $105_{-14}^{+51}$ & $81_{-1}^{+82}$ & Corona temperature \\
\hline$R_{\text {cor }}\left(r_{\mathrm{g}}\right)$ & $59_{-19}^{+60}$ & $10_{-6}^{+38}$ & $20_{-9}^{+41}$ & $10_{-6}^{+9}$ & Corona normalization radius \\
\hline$L_{p}\left(\operatorname{erg~s}^{-1}\right)$ & $4.3 \times 10^{38}$ & $5.1 \times 10^{38}$ & - & - & Accelerated proton power \\
\hline$L_{e}\left(\mathrm{erg} \mathrm{s}^{-1}\right)$ & $1.3 \times 10^{36}$ & $1.9 \times 10^{36}$ & $2.1 \times 10^{36}$ & $3.3 \times 10^{36}$ & Accelerated electron power \\
\hline$E_{p, \max }(\mathrm{eV})$ & $2.7 \times 10^{15}$ & $1.8 \times 10^{15}$ & - & - & Proton maximum energy \\
\hline$E_{e, \max }(\mathrm{eV})$ & $6.1 \times 10^{10}$ & $2.2 \times 10^{10}$ & $3.3 \times 10^{10}$ & $8.8 \times 10^{10}$ & (primary) Electron maximum energy \\
\hline$\chi^{2} / \mathrm{DoF}$ & $9597.8 / 2439$ & $2451.9 / 2439$ & $4064.7 / 2439$ & $2237.1 / 2439$ & $\chi^{2} /$ degrees of freedom \\
\hline
\end{tabular}
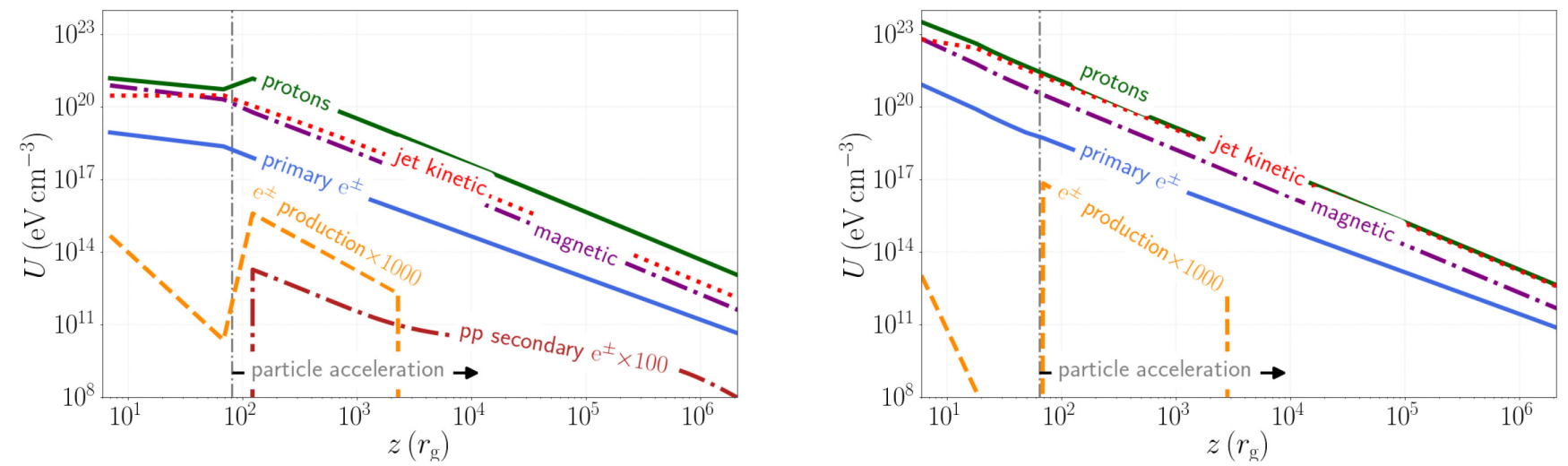

Figure 1. Contributions to the total energy density as a function of the distance along the jet for the model with a power-law index $p=1.7$, for the lepto-hadronic case (left) and the purely leptonic case (right). The particle acceleration initiates at the vertical dot-dashed grey line. The jump in the proton energy density on the left plot is due to proton acceleration. We do not assume extraction of energy from other components to accelerate the particles. The proton and the jet kinetic energy density of the right plot coincide because no proton acceleration is taken into account. We stop to calculate the pair production after some distance because it has insignificant contribution.

density of the magnetic field is higher than the energy density of the (primary) electrons. We also show the energy density of the secondary pairs due to photon annihilation. We see that this process has its peak but still insignificant contribution in jet segments of high compactness, i.e. high photon number density at the jet base and in the particle acceleration region. The number density of the target photons drops significantly after the jet base, which suppresses the pair production. At the particle acceleration region the compactness increases due to the non-thermal synchrotron and SSC photons. For the case of the lepto-hadronic model, we also show the energy density of secondary electrons from pp interactions, even though their energy density is more than five orders of magnitude lower than the rest.

\subsection{Best fits to the multiwavelength spectrum}

The combined data of Cyg $\mathrm{X}-1$ presented in Section 2 result in a broad-band spectrum covering almost 15 orders of magnitude in photon frequency. We are able to reasonably fit all wavebands simultaneously with our model. Figs 2 and 3 show all four different model scenarios. The residuals are not always negligible, especially for the X-ray spectrum between $10^{17}$ and $10^{19} \mathrm{~Hz}$. This is a natural consequence of our broad-band fit. The superb data coverage of the $\mathrm{X}$-rays suggests a number of specific spectral features, e.g. due to relativistic reflection off the inner accretion disc, which our oversimplified model for the corona is not able to describe in detail. Such an in-depth treatment of all X-ray features is outside the scope of this 

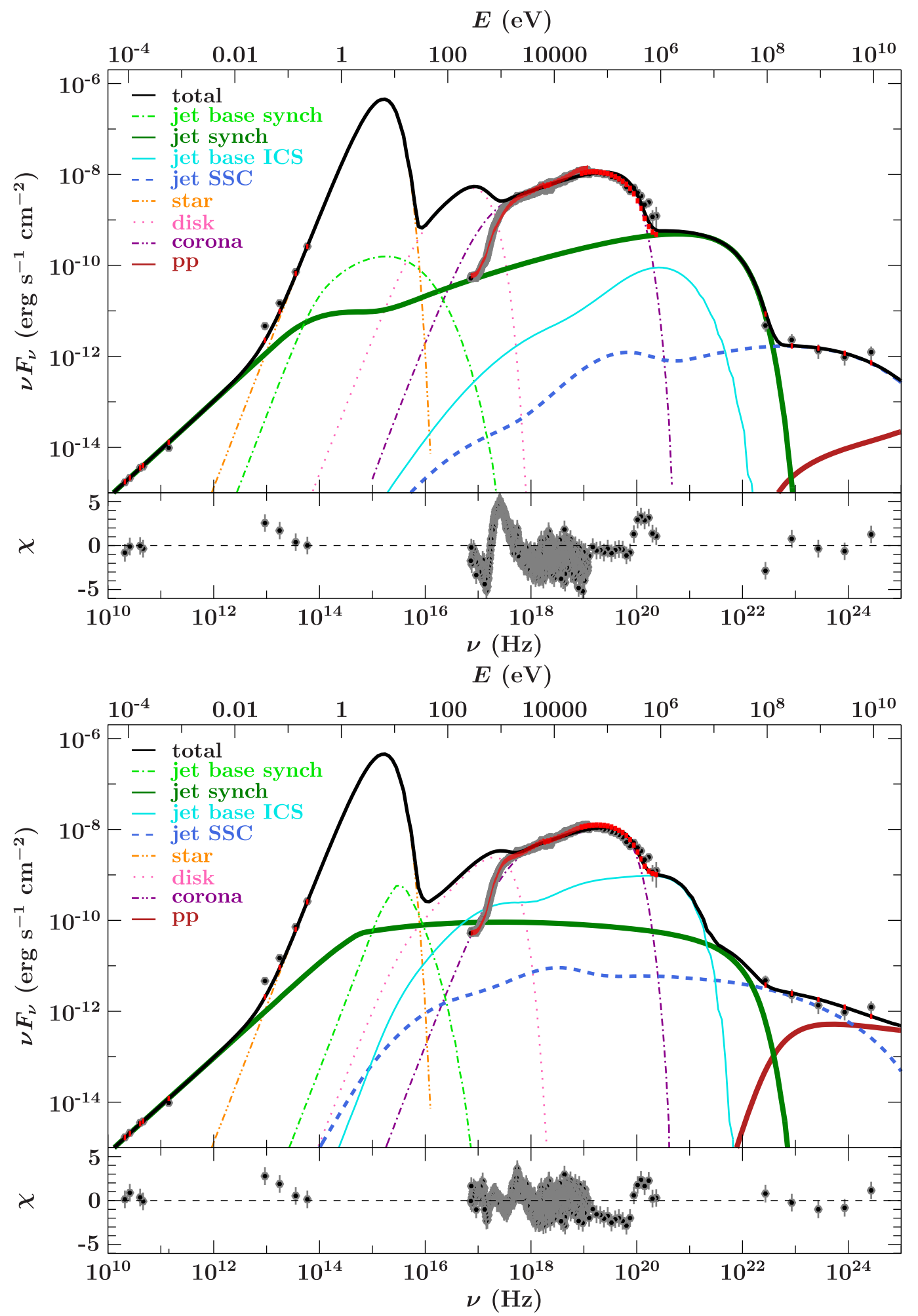

Figure 2. The best-fitting multiwavelength spectrum of Cyg X-1 for the two lepto-hadronic scenarios with $p=1.7$ (top) and $p=2.2($ bottom) and their $\chi$ residuals. The solid black line shows the total unabsorbed spectrum. The absorbed spectrum that we fitted to the data in detector space is shown as solid red line. We also show some individual unabsorbed model components, i.e. the broad-band radio-to-gamma-ray synchrotron spectrum from primary electrons (thick solid green line), the ICS spectrum ranging from $\mathrm{eV}$ to $\mathrm{GeV}$ (dashed dark blue line), the pp spectral component arising from the neutral pion decay (solid red line), disc photons upscattered in the thermal corona (dotted-dashed purple line), the blackbody component emitted by the companion star (double-dotted-dashed orange line), and the multi-temperature thermal spectrum arising from the accretion disk (dotted magenta line). The dotted-dashed light green line shows the synchrotron radiation from thermal electrons and the triple-dotted-dashed light blue line shows the ICS from regions before the particle acceleration region. In the case where $p=1.7$ the jet-synchrotron dominates in the $\mathrm{MeV}$ band explaining the high degree of reported linear polarization. In the soft case of $p=2.2$, the fit does not explain the reported polarization. 

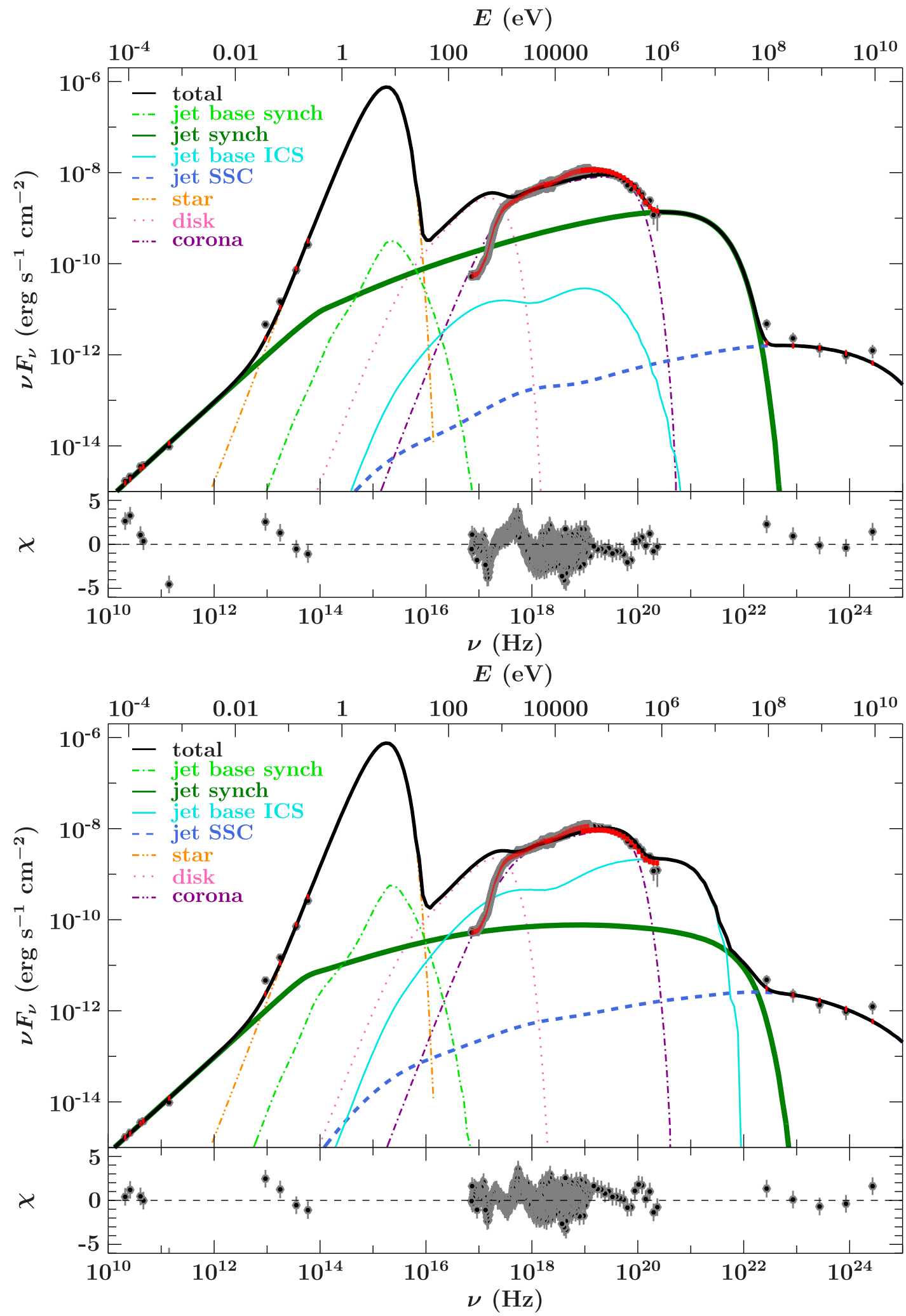

Figure 3. Similar to Fig. 2 but for the two leptonic scenarios with $p=1.7$ (top) and $p=2.2$ (bottom).

work (see e.g. Tomsick et al. 2013; Parker et al. 2015; Duro et al. 2016; Basak et al. 2017).

We also take into account synchrotron-self absorption in the radio band and photoabsorption of X-ray photons with the column density
$N_{H}=0.6 \times 10^{22} \mathrm{~cm}^{-2}$ (Grinberg et al. 2015). The wind of the companion star could in principle attenuate the radio band even at inferior conjunction (when the companion star is behind the jet on the line of sight) examined here. Nevertheless, the $20 \mathrm{GHz}$ radio 


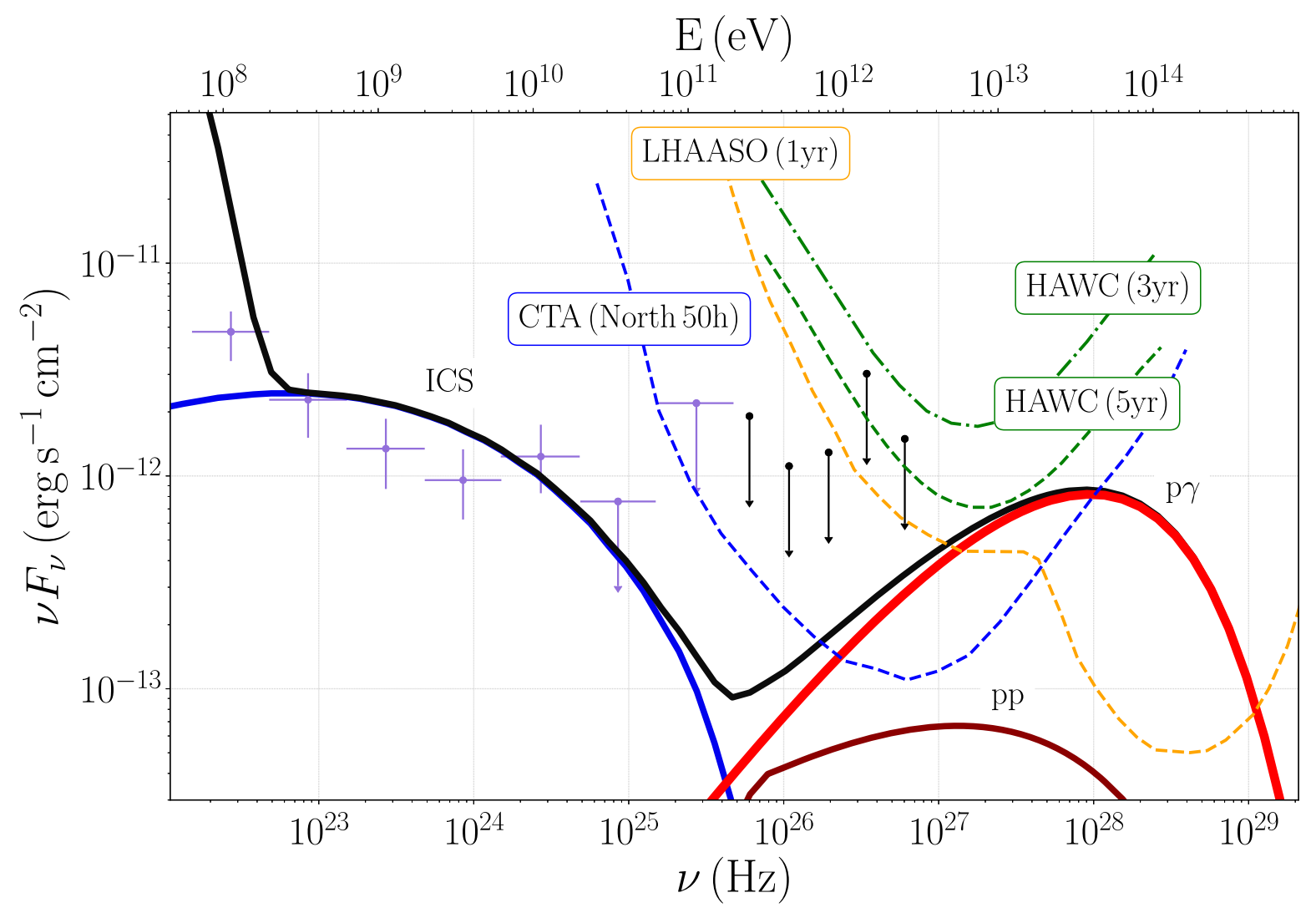

Figure 4. The GeV-to-TeV regime of the multiwavelength spectrum of Cyg X-1 for the lepto-hadronic scenario with $p=1.7$. The black line shows the total spectrum. The ICS (solid dark blue) explains the Fermi/LAT (purple) data points in the GeV band. The neutral pion decay from $\mathrm{p} \gamma$ (thick light red) dominates the pp (dark red) and peaks in the TeV regime. Such emission will be detectable by future generation facilities, such as the CTA (dashed blue, adopted from www.cta-observatory.org), and LHAASO (dashed orange, adopted from Bai et al. 2019). We also plot for comparison the upper limits of MAGIC (black upper limits) from Ahnen et al. (2017), and the 3 (dot-dashed green) and 5-yr (dashed green) sensitivity of HAWC adopted from Abeysekara et al. (2013).

emission originates from a region much further out in the jets than 10 times the separation of the system so this attenuation should be insignificant (see e.g. Szostek \& Zdziarski 2007).

\subsection{GeV-TeV spectrum}

The lepto-hadronic model with $p=1.7$ is the only one that predicts significant $\mathrm{TeV}$ emission. In Fig. 4, we plot the $\mathrm{GeV}$ to $\sim \mathrm{PeV}$ regime of its multiwavelength spectrum. For a comparison, we add the upper limits of the Major Atmospheric Gamma Imaging Cherenkov Telescopes - MAGIC (Ahnen et al. 2017), the 3 and 5yr sensitivity of the High-Altitude Water Cherenkov Observatory HAWC (Abeysekara et al. 2013), and the predicted sensitivity of the Cherenkov Telescope Array - CTA (from www.cta-observatory.org) and of the Large High Altitude Air Shower Observatory - LHAASO (Bai et al. 2019).

In the $\mathrm{GeV}$ range, we did not take into account photon annihilation due to the stellar photon field because the data we consider here are taken while the source was in the inferior conjunction. Further GeV observations will help to better understand the orbital modulation of Cyg $\mathrm{X}-1$ as well in this domain.

Our evaluated spectrum above $0.1 \mathrm{TeV}\left(10^{25} \mathrm{~Hz}\right)$ is dominated by the gamma-rays produced via neutral pion decay from the hadronic collisions. The dominant process at the highest photon energies is the $\mathrm{p} \gamma$ interaction, between accelerated jet protons and the synchrotron $\mathrm{MeV}$ photons. The number density of other target photon fields is negligible compared to this $\mathrm{MeV}$ band in the jet rest frame. The flux levels predicted by our model are overall higher than the sensitivity limits of next-generation gamma-ray telescopes. HAWC, LHAASO, and CTA will therefore be key for breaking further degeneracies within our model, and constraining important processes such as the $\mathrm{p} \gamma$ interactions in astrophysical jets.

For our discussion of the highest energies, we only consider the hard lepto-hadronic model $(p=1.7)$, as the soft model $(p=2.2)$ cannot explain the $\mathrm{MeV}$ polarization. Neither leptonic model can produce any $\mathrm{TeV}$ emission via ICS, because the electron scattering with $\mathrm{GeV}$ photons occurs deep in the Klein-Nishina regime. Thus, no further order scatters can occur inside the jets that would produce significant $\mathrm{TeV}$ radiation. A solid $\mathrm{TeV}$ detection would therefore rule out the leptonic models.

\section{DISCUSSION}

A key open issue regarding Cyg $\mathrm{X}-1$ is the polarised $0.4-2 \mathrm{MeV}$ tail detected by INTEGRAL while the source is in the hard state (Laurent et al. 2011; Jourdain et al. 2012; Rodriguez et al. 2015; Cangemi et al. 2020). The above studies all independently conclude that the linear polarization degree of the $\mathrm{MeV}$ emission is of the order of 50-70 per cent. While there is an overall agreement on the degree of polarization, INTEGRAL does not have the spatial resolution to resolve the source, thus the integrated polarization angle over the entire system does not provide constraining information on the detailed magnetic field geometry of the source. 
Such high degree of polarization, requires a structured and well-ordered magnetic field. High-resolution numerical simulations suggest that the wind of the accretion disc, which is associated to the corona, is very turbulent and could not explain such structured magnetic field (Liska et al. 2017; Chatterjee et al. 2019; Liska, Tchekhovskoy \& Quataert 2020). Hence, jet-synchrotron is more likely to explain the $\mathrm{MeV}$ polarization.

In this work, we take advantage of the new and unprecedented (in broadband simultaneity) CHOCBOX multiwavelength data set to revisit the question of leptonic versus hadronic processes, using a more sophisticated multizone approach. In particular we explore the consequences of taking the $\mathrm{MeV}$ polarization as a 'hard' constraint, and the consequences for potential $\mathrm{TeV}$ gamma-ray emission. We find that the only way to produce sufficient synchrotron flux to fit the $\mathrm{MeV}$ data is by assuming a hard power-law distribution of accelerated electrons with $p=1.7$. If we assume a soft power-law with $p=2.2$ we fail to match this constraint.

These two different power-law indices of 1.7 and 2.2 are typically associated with different particle acceleration mechanisms. The hard particle spectrum $(p=1.7)$ suggests second-order Fermi acceleration (e.g. Rieger et al. 2007) or magnetic reconnection (e.g. Biskamp 1996; Sironi \& Spitkovsky 2014; Petropoulou \& Sironi 2018 or Khiali, de Gouveia Dal Pino \& del Valle 2015 for the case of Cyg X-1 specifically). The softer injection value of $p=2.2$ is more suggestive of non-linear diffusive shock acceleration (e.g. Drury 1983; Malkov \& Drury 2001; Caprioli 2012), but we show that the high degree of $\mathrm{MeV}$ polarization cannot be attained. We find that the best fits to the data require a more efficient acceleration mechanism to be the dominant source of non-thermal particles. We note however that when we define the acceleration timescale to derive the maximum energy of the particles (see equations 10 and 9), we use a simplified expression that is commonly used to describe firstorder Fermi acceleration. In future work, we will include energy dependence to the acceleration timescale to explore in detail the different acceleration mechanisms.

Taking as a constraint the explanation of both the observed $\mathrm{MeV}$ spectrum and the $\mathrm{GeV} \gamma$-rays, we require a generally high particle acceleration efficiency $f_{\text {sc }}$. For the models with a soft particle spectrum, we require a higher efficiency $(0.1)$ as opposed to the models with the hard particle spectrum, where an acceleration efficiency of 0.01 is sufficient. This parameter also drives the maximum achievable energy of the particles. We find a maximum electron energy of 10$100 \mathrm{GeV}$ (see Table 3) and proton energy of $\sim 10^{15} \mathrm{eV}$. The high particle energies we find for both electrons and protons translate to a required high total power in particles, i.e. $\sim 10^{36} \mathrm{erg} \mathrm{s}^{-1}$ for electrons and $\sim 10^{39} \mathrm{erg} \mathrm{s}^{-1}$ for protons.

Independent measurements of the total kinetic jet power are useful to benchmark our fitted values for the total injected energy. One can estimate the jet power from the bubble-like structure located $5 \mathrm{pc}$ from Cyg X-1 caused by the apparent interaction between the jet and the ISM. The mechanical power required to inflate such bubble has been calculated to be of the order of $10^{37} \mathrm{erg} \mathrm{s}^{-1}$ (Gallo et al. 2005). It is, however, still debated whether the jet is solely evacuating this bubble, or whether other feedback channels, such as the companion star's stellar wind, play a role. In that case, the jet power estimated by Gallo et al. (2005) would have to be considered as an upper limit (Sell et al. 2014). This estimate would lead to the exclusion of the lepto-hadronic model because of its exceeding jet power, while the purely leptonic model requires merely 10 per cent of the estimated power. This large discrepancy (up to 3 order of magnitude) driven by the inclusion/exclusion of hadronic processes is a well-known issue in the field (e.g. Bosch-Ramon, Khangulyan \& Aharonian 2008;
Zdziarski et al. 2012; Malyshev et al. 2013; Zdziarski et al. 2014; Zhang, Xu \& Lu 2014; Pepe et al. 2015; Zdziarski et al. 2017; Beloborodov 2017; Fernández-Barral et al. 2017).

Most hadronic models show jet powers close to Eddington limit either for Galactic or extragalactic sources (Böttcher et al. 2013; Zdziarski \& Böttcher 2015). However, there are a few possible ways of extracting further power from the system to the particles without violating other constraints. One possibility is a much more efficient dissipation of either magnetic or kinetic energy via particle acceleration, i.e. greater than 10 per cent. Another, perhaps more likely scenario is the one where the jets are launched by a magnetically dominated (MAD) accretion flow and a spinning black hole. In such systems, the jet can benefit from an efficient extraction of power both from the accretion disc and the black hole rotation (Blandford \& Znajek 1977; Narayan, Igumenshchev \& Abramowicz 2003; Tchekhovskoy, Narayan \& McKinney 2011). Alternatively, the total proton power can be reduced. One possibility is that the jets are predominantly leptonic up to when the bulk flow is accelerated to maximum velocity. The majority of protons are then mass-loaded further away from the launching point either by the wind of the accretion disc or of the companion star (Chatterjee et al. 2019; Perucho 2020). To calculate the total proton power in this work, we sum the proton power per segment along the jet. If we assume that protons accelerate only within a small part of the jet, then the total power could be significantly reduced (Pepe et al. 2015; Khiali et al. 2015; Abeysekara et al. 2018). Such assumptions would however only increase the free parameters of our model. Therefore, we decided to restrict ourselves to 'standard' assumptions for fitting the data, and to ease comparison with prior approaches.

\subsection{Comparison with previous works}

In Table 4, we present a schematic comparison between the main features of our new model and of a sample of similar works used to explain the multiwavelength spectrum of Cyg X-1. The models that we consider here are the following: Romero et al. 2014 (R14), Zdziarski et al. 2014 (Z14), Khiali et al. 2015 (K15), Pepe et al. 2015 (P15), and Zdziarski et al. 2017 (Z17).

It is generally agreed that the radio-to-FIR spectrum of Cyg $\mathrm{X}-1$ is produced by its relativistic jets, and likely the $\mathrm{GeV}$ emission as well. Numerous studies dedicated to fitting high signal-to-noise ratio X-ray spectra of Cyg X-1 invoke the presence of a corona with hot, thermal electrons to upscatter soft disc photons up to $\sim 100 \mathrm{keV}$ energies, as this is standard for most XRB hard-state models (Tomsick et al. 2013; Parker et al. 2015; Duro et al. 2016; Basak et al. 2017; Walter \& Xu 2017). Furthermore, the companion of Cyg $X-1$ is a high-mass donor star; hence, an additional blackbody (or even a more detailed stellar model) spectral component is required.

The key differences between approaches centre primarily on the nature of the particle acceleration in the jets, the role of the jets at high energies, and the level of detail in the modelling of the jet properties.

Constraining the contribution of the jets at high energies, and thus the total power requirements, hinges on the $\mathrm{MeV}$ polarization and the gamma-rays. Many of the prior works did not consider the $\mathrm{MeV}$ polarization as a hard constraint. For those that did, R14 suggest that the synchrotron radiation from secondary electrons in the corona could explain the $\mathrm{MeV}$ tail. As we discussed above though, jet synchrotron is a more likely origin. Z14 explain the MeV flux as a result of jet synchrotron from primary electrons. They presented only a purely leptonic model and thus no $\mathrm{TeV}$ detection can be predicted. This choice thus places them in a regime with reasonable 


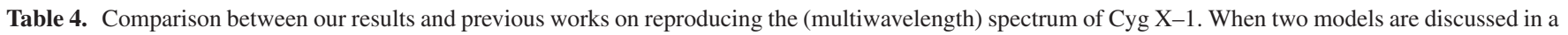

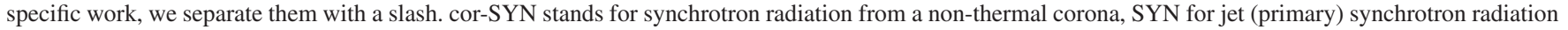

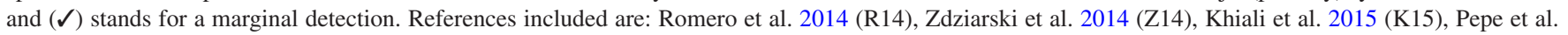
2015 (P15), and Zdziarski et al. 2017 (Z17).

\begin{tabular}{|c|c|c|c|c|c|c|c|}
\hline \multirow[b]{2}{*}{ Features/model } & \multicolumn{5}{|c|}{ Other works } & \multicolumn{2}{|c|}{ This work } \\
\hline & $\mathrm{R} 14$ & Z14 & K15 & $\mathrm{P} 15$ & $\mathrm{Z} 17$ & Hadronic & Leptonic \\
\hline Power-law index ${ }^{a}$ & 2.2 & $1.4 / 2.5$ & 1.8 & $2.0 / 2.4$ & 2.2 & $1.7 / 2.2$ & $1.7 / 2.2$ \\
\hline Corona presence & $\checkmark$ & $\checkmark$ & $x$ & $\checkmark$ & $\checkmark$ & $\checkmark$ & $\checkmark$ \\
\hline Hadronic processes & $\checkmark$ & $x$ & $\checkmark$ & $\checkmark$ & $x$ & $\checkmark$ & $x$ \\
\hline Simultaneous data & $x$ & $x$ & $x$ & $x$ & $x$ & $\checkmark$ & $\checkmark$ \\
\hline Statistical modelling/MCMC & $x$ & $x$ & $x$ & $x$ & $x$ & $\checkmark$ & $\checkmark$ \\
\hline $\mathrm{MeV} \mathrm{X}$-rays origin & cor-SYN & SYN/COM & SYN & SYN/COM & $\mathrm{COM}$ & SYN/COM & SYN/COM \\
\hline Explain $\mathrm{MeV}$ polarization & $\checkmark$ & $\sqrt{ } / X$ & $x$ & $\sqrt{ } / X$ & $x$ & $\sqrt{ } / X$ & $\sqrt{ } / X$ \\
\hline CTA@TeV prediction & $x$ & $x$ & $\checkmark$ & $\sqrt{ } / X$ & $x$ & $\checkmark$ & $x$ \\
\hline LHAASO@100TeV prediction & $x$ & $x$ & $(\checkmark)$ & $(\checkmark) / x$ & $x$ & $\checkmark$ & $x$ \\
\hline
\end{tabular}

Note. ${ }^{a}$ Accelerated particle power-law index $p: N(E) \propto E^{-p}$.

total jet powers. P15 manage to reproduce the MeV tail in a leptohadronic scenario with primary electron synchrotron radiation. This is similar to our lepto-hadronic model with $p=1.7$ but they use a much softer injected electron distribution. They manage to restrict the total proton power by making two assumptions discussed also above: first, protons are accelerated only from a minimum Lorentz factor of $\gamma_{p, \min }=100$ and second, the particle acceleration terminates at some distance from the jet base. None of these works though attempted to fit their free parameters to simultaneous data and perform statistical analysis, which may affect their conclusions.

\subsection{Perspective for CTA, HAWC, and LHAASO}

In Fig. 4, we compare the results of the lepto-hadronic model with $p=1.7$ to the upper limits set by MAGIC after almost $100 \mathrm{hr}$ of observations (Ahnen et al. 2017). In addition, HAWC released its second catalogue of $\mathrm{TeV}$ sources and a catalogue of 9 Galactic sources after $1000 \mathrm{~d}$ of operation, but Cyg X-1 was not included in either of them (Abeysekara et al. 2017; Abeysekara et al. 2020 , respectively). Thus, we also plot the sensitivity predicted by the HAWC collaboration for $5 \mathrm{yr}$ of operation (Abeysekara et al. 2013).

We plot the predicted sensitivity of CTA for TeV gamma-rays, as well as the sensitivity of LHAASO (Bai et al. 2019), which mostly focuses on $\sim 100 \mathrm{TeV}$. In the hadronic model with $p=$ 1.7, the $\mathrm{TeV}$ emission is dominated by the $\mathrm{p} \gamma$ inelastic collisions between accelerated protons and synchrotron photons of the jet. The peak is at $20 \mathrm{TeV}$ and the corresponding flux is expected to be $2 \times 10^{-12} \mathrm{erg} \mathrm{cm}^{-2} \mathrm{~s}^{-1}$, significantly above the predicted CTA sensitivity for $50 \mathrm{hr}$ of observation from the north site. Moreover, the spectral index of this $\mathrm{TeV}$ emission is predicted to be positive and $\sim 0.5$ for energies between 0.1 and $10 \mathrm{TeV}$ (i.e. $F_{\nu} \propto \nu^{0.5}$ ).

An interesting aspect of our model is that the photomeson interactions dominate the pp collisions. The energy threshold of $\mathrm{pp}$ inelastic collisions, in general, is lower than $\mathrm{p} \gamma$. Nevertheless, the number density of the target protons from the thermal wind of the companion star within the jet is constant up to $z \simeq a_{\star}$ regardless of the physics of the jets (see equation 15). On the other hand, the number density of the target photons of $\mathrm{p} \gamma$ are highly model-dependent. For the hadronic models presented here the dominant target photons are the synchrotron photons of each jet segment. Consequently, in the case of the hard particle distribution $(p=1.7)$ where the energy density of $\mathrm{MeV}$ photons is ten times higher than that of the soft particle distribution (see Fig. 2), the $\mathrm{p} \gamma$ process dominates the $\mathrm{TeV}$ band.

A detection of $\mathrm{TeV}$ photons and a measurement of the spectral index of this emission by forthcoming very high-energy facilities could therefore give further insights into the acceleration mechanism. Finally, regardless of the spectral shape, the detection of Cyg X-1 from HAWC, and especially from CTA or LHAASO would exclude the possibility of purely leptonic jets for this source.

\section{SUMMARY AND CONCLUSIONS}

In this work, we present a new multizone jet model, based on the initial work of Markoff et al. (2005) and references above. We implement proton acceleration and inelastic hadronic collisions (proton-proton and proton-photon, Kelner et al. 2006; Kelner \& Aharonian 2008, respectively). We include the distributions of secondary electrons and gamma-rays produced through pion decay. We further improve the existing leptonic processes with more sophisticated pair-production calculations (Coppi \& Blandford 1990; Böttcher \& Schlickeiser 1997), as well as take into account the proper geometry of the companion star as seen in the jet rest frame. With such enhancements, we can make more accurate predictions of the high energy phenomena related to astrophysical jets, particularly the nonthermal emitted radiation.

Along with this new model, we present the first broadband, simultaneous data set obtained by the CHOCBOX campaign for Cyg X-1 (Uttley 2017). This data set covers ten orders of magnitude in photon energy, from radio wavelengths to MeV X-rays. These bands are most susceptible to faster variability and hence simultaneous high-quality observations are beneficial to break model degeneracies.

The keV-to-MeV spectrum of Cyg X-1 exhibits significant evidence of linear polarization. The $\mathrm{keV}$ spectrum shows low degree of linear polarization (Chauvin et al. 2018a, b) but the 0.4 $2 \mathrm{MeV}$ is highly polarized at a level of $50-70$ per cent (Laurent et al. 2011; Jourdain et al. 2012; Rodriguez et al. 2015; Cangemi et al. 2020). We interpret this high degree of linear polarization in the $\mathrm{MeV}$ band as synchrotron radiation emitted by (primary) electrons accelerated inside the jets of Cyg $X-1$ in the presence of a highly ordered magnetic field. Such non-turbulent, dynamically dominant magnetic fields are most likely associated with astrophysical jets. To achieve the required $\mathrm{MeV}$ synchrotron flux, we must inject a hard power law of accelerated electrons with index of $p=1.7$. 
We investigate the implications of the above assumptions for a purely leptonic and a lepto-hadronic scenario, performing statistical analyses to find the best fits to the CHOCBOX data set. Using an MCMC approach, we explore the parameter phase-space in order to constrain the parameters and minimize degeneracy. This paper is the first to compare a purely leptonic to a lepto-hadronic model for the case of XRB jets based on statistical analysis.

We find that the jet geometry does not significantly differ between the two compared scenarios; the main differences are the $\mathrm{TeV}$ radiation and the power requirements. Only the hadronic model is capable of producing significant $\mathrm{TeV}$ emission detectable by the next generation gamma-ray telescopes of HAWC, LHAASO, and CTA. Interestingly, we find that the dominant hadronic process is the proton-photon interaction. This scenario however requires nearEddington power in the accelerated protons, using the most basic assumptions. We discuss ways around this issue but leave that for future work, in the case of a TeV detection. Such a detection would be a game-changer for the field of XRBs, and support the possibility that Galactic CRs originate in more sources than only supernovae.

\section{ACKNOWLEDGEMENTS}

We would like to thank the reviewer for the very helpful comments on improving the original manuscript. DK would like to thank Maria Petropoulou for fruitful discussions, and Ping Zhou and Thomas Russell for useful comments on the manuscript. DK, SM, ML, and AC were supported by the Netherlands Organisation for Scientific Research (NWO) VICI grant (no. 639.043.513). VG is supported through the Margarete von Wrangell fellowship by the ESF and the Ministry of Science, Research and the Arts BadenWürttemberg. JCAM-J is the recipient of an Australian Research Council Future Fellowship (FT140101082), funded by the Australian government. This work is based on observations carried out under the project number W15BQ with the IRAM NOEMA Interferometer. IRAM is supported by INSU/CNRS (France), MPG (Germany) and IGN (Spain). This research made use of ASTROPY (http: //www.astropy.org) a community-developed core PYTHON package for Astronomy (Astropy Collaboration et al. 2013; Price-Whelan et al. 2018), MATPLOTLIB (Hunter 2007), NUMPY (Oliphant 2006), SCIPY (Virtanen et al. 2020), ISIS functions (ISISscripts) provided by ECAP/Remeis observatory and MIT (http://www.sternwarte.u nierlangen.de/isis/), and the CTA instrument response functions provided by the CTA Consortium and Observatory, see http://www.ct a-observatory.org/science/cta-performance/ (version prod3b-v2) for more details.

\section{DATA AVAILABILITY}

The data underlying this article are available in Zenodo, at https: //dx.doi.org/10.5281/zenodo.4126910.

\section{REFERENCES}

Abeysekara A. et al., 2013, Astropart. Phys., 50-52, 26

Abeysekara A. U. et al., 2017, ApJ, 843, 40

Abeysekara A. U. et al., 2018, Nature, 562, 82

Abeysekara A. et al., 2020, Phys. Rev. Lett., 124, 021102

Aharonian F., 2000, New Astron., 5, 377

Aharonian F., Yang R., de Oña Wilhelmi E., 2019, Nature Astron., 3, 561

Aharonian F. A., , 2004, Very High Energy Cosmic Gamma Radiation: A Crucial Window on the Extreme Universe. World Scientific, Singapore Aharonian F. A., 2002, MNRAS, 332, 215
Ahnen M. L. et al., 2017, MNRAS, 472, 3474

Astropy Collaboration et al., 2013, A\&A, 558, A33

Axford W., 1969, Invited Papers. Springer, Berlin, Heidelberg, p. 155

Bai X. et al., 2019, preprint (arXiv:1905.02773)

Basak R., Zdziarski A. A., Parker M., Islam N., 2017, MNRAS, 472, 4220

Beloborodov A. M., 2017, ApJ, 850, 141

Bird D. J. et al., 1993, Phys. Rev. Lett., 71, 3401

Biskamp D., 1996, Astrophys. Space Sci., 242, 165

Blandford R. D., Königl A., 1979, ApJ, 232, 34

Blandford R. D., Ostriker J. P., 1978, ApJ, 221, L29

Blandford R. D., Znajek R. L., 1977, MNRAS, 179, 433

Blumenthal G. R., Gould R. J., 1970, Rev. Mod. Phys., 42, 237

Bodaghee A., Tomsick J. A., Pottschmidt K., Rodriguez J., Wilms J., Pooley G. G., 2013, ApJ, 775, 98

Bolton C. T., 1972, Nature, 235, 271

Bosch-Ramon V., Khangulyan D., Aharonian F. A., 2008, A\&A, 489, L21

Bosch-Ramon V., Romero G. E., Paredes J. M., 2006, A\&A, 447, 263

Brown A. et al., 2018, A\&A, 616, A1

Böttcher M., Dermer C. D., 2005, ApJ, 634, L81

Böttcher M., Reimer A., Sweeney K., Prakash A., 2013, ApJ, 768, 54

Böttcher M., Schlickeiser R., 1997, A\&A, 325, 866

Cangemi F. et al., 2020, submitted

Caprioli D., 2012, JCAP, 07, 038

Chatterjee K., Liska M., Tchekhovskoy A., Markoff S. B., 2019, MNRAS, 490, 2200

Chauvin M. et al., 2018a, Nature Astron., 2, 652

Chauvin M. et al., 2018b, MNRAS, 483, L138

Cooper A. J., Gaggero D., Markoff S., Zhang S., 2020, MNRAS, 493, 3212

Coppi P. S., Blandford R. D., 1990, MNRAS, 245, 453

Crumley P., Caprioli D., Markoff S., Spitkovsky A., 2019, MNRAS, 485, 5105

Crumley P., Ceccobello C., Connors R. M. T., Cavecchi Y., 2017, A\&A, 601, A87

Drury L. O., 1983, Rep. Progr. Phys., 46, 973

Duro R. et al., 2016, A\&A, 589, A14

Díaz Trigo M., Miller-Jones J. C. A., Migliari S., Broderick J. W., Tzioumis T., 2013, Nature, 504, 260

Eichmann B., Rachen J., Merten L., van Vliet A., Tjus J. B., 2018, J. Cosmol. Astropart. Phys., 2018, 036

Ellison D. C., Jones F. C., Reynolds S. P., 1990, ApJ, 360, 702

Fabrika S., 2004, Astrophys. Space Phys. Rev., 12, 1

Falcke H., Biermann P. L., 1995, A\&A, 293, 665

Fender R. P., Maccarone T. J., van Kesteren Z., 2005, MNRAS, 360, 1085

Fender R. P., Stirling A. M., Spencer R. E., Brown I., Pooley G. G., Muxlow T. W. B., Miller-Jones J. C. A., 2006, MNRAS, 369, 603

Fernández-Barral A. et al., 2017, Proc.Sci., Gamma rays from microquasars Cygnus X-1 and Cygnus X-3. SISSA, Trieste, PoS(ICRC2017)734

Gaisser T. K., Engel R., Resconi E., 2016, Cosmic Rays and Particle Physics. Cambridge Univ. Press, Cambridge

Gallo E., Fender R., Kaiser C., Russell D., Morganti R., Oosterloo T., Heinz S., 2005, Nature, 436, 819

Gandhi P., Rao A., Johnson M. A. C., Paice J. A., Maccarone T. J., 2019, MNRAS, 485, 2642

Giannios D., 2010, MNRAS, 408, L46

Gies D. R. et al., 2008, ApJ, 678, 1237

Grinberg V. et al., 2015, A\&A, 576, A117

Hada K. et al., 2016, ApJ, 817, 131

Harrison F. A. et al., 2013, ApJ, 770, 103

Heinz S., Sunyaev R., 2002, A\&A, 390, 751

Hillas A. M., 1984, ARA\&A, 22, 425

Hjellming R. M., Johnston K. J., 1988, ApJ, 328, 600

Hjellming R. M., Rupen M. P., 1995, Nature, 375, 464

Houck J. C., Denicola L. A., 2000, in Manset N., Veillet C., Crabtree D., eds, ASP Conf. Ser. Vol. 216, Astronomical Data Analysis Software and Systems IX. Astron. Soc. Pac., San Francisco, p. 591 
Hunter J. D., 2007, Comput. Sci. Eng., 9, 90

Jokipii J., 1987, ApJ, 313, 842

Jourdain E., Roques J., Chauvin M., Clark D., 2012, ApJ, 761, 27

Kafexhiu E., Aharonian F., Taylor A. M., Vila G. S., 2014, Phys. Rev. D, 90, 123014

Kelner S., Aharonian F., 2008, Phys. Rev. D, 78, 034013

Kelner S., Aharonian F. A., Bugayov V., 2006, Phys. Rev. D, 74, 034018

Khiali B., de Gouveia Dal Pino E. d., del Valle M. V., 2015, MNRAS, 449, 34

Kulikov G., Khristiansen G., 1959, Sov. Phys. JETP, 35, 441

Laurent P., Rodriguez J., Wilms J., Cadolle Bel M., Pottschmidt K., Grinberg V., 2011, Science, 332, 438

Lebrun F. et al., 2003, A\&A, 411, L141

Liska M., Hesp C., Tchekhovskoy A., Ingram A., van der Klis M., Markoff S., 2017, MNRAS, 474, L81

Liska M., Tchekhovskoy A., Quataert E., 2020, MNRAS, 494, 3656

Lister M. L. et al., 2013, AJ, 146, 120

Liu R.-Y., Rieger F., Aharonian F., 2017, ApJ, 842, 39

Lucchini M., Markoff S., Crumley P., Krauß F., Connors R. M. T., 2019, MNRAS, 482, 4798

Lucchini M., Russell T. D., Markoff S. B., Vincentelli F., Gardenier D., Ceccobello C., Uttley P., 2020, submitted

Maitra D., Markoff S., Brocksopp C., Noble M., Nowak M., Wilms J., 2009, MNRAS, 398, 1638

Malkov M., Drury L. O., 2001, Rep. Progr. Phys., 64, 429

Malyshev D., Zdziarski A. A., Chernyakova M., 2013, MNRAS, 434, 2380

Mannheim K., Schlickeiser R., 1994, A\&A, 286, 983

Markoff S., Falcke H., Fender R., 2001, A\&A, 372, L25

Markoff S., Nowak M. A., Wilms J., 2005, ApJ, 635, 1203

Marscher A. P. et al., 2008, Nature, 452, 966

Mastichiadis A., Kirk J. G., 2002, PASA, 19, 138

Mastroserio G., Ingram A., van der Klis M., 2019, MNRAS, 488, 348

McMullin J. P., Waters B., Schiebel D., Young W., Golap K., 2007, in Shaw R. A., Hill F., Bell D. J., eds, Conf. Ser., Vol. 376, Astronomical Data Analysis Software and Systems XVI. Astron. Soc. Pac., San Francisco, p. 127

Miller-Jones J. C. A. et al., 2020, submitted

Miller J. M., Wojdowski P., Schulz N. S., Marshall H. L., Fabian A. C., Remillard R. A., Wijnands R., Lewin W. H. G., 2005, ApJ, 620, 398

Mioduszewski A. J., Rupen M. P., Hjellming R. M., Pooley G. G., Waltman E. B., 2001, ApJ, 553, 766

Mirabel I. F., Rodríguez L. F., 1994, Nature, 371, 46

Mücke A., Protheroe R., Engel R., Rachen J., Stanev T., 2003, Astropart. Phys., 18, 593

Narayan R., Igumenshchev I. V., Abramowicz M. A., 2003, Publ. Astron. Soc. Japan, 55, L69

Oliphant T. E., 2006, A Guide to NumPy, Vol. 1. Trelgol Publishing, USA

Orosz J. A., McClintock J. E., Aufdenberg J. P., Remillard R. A., Reid M. J., Narayan R., Gou L., 2011, ApJ, 742, 84

Parker M. L. et al., 2015, ApJ, 808, 9

Pepe C., Vila G. S., Romero G. E., 2015, A\&A, 584, A95

Perucho M., 2020, MNRAS, 494, L22

Petropoulou M., Sironi L., 2018, MNRAS, 481, 5687
Price-Whelan A. M. et al., 2018, AJ, 156, 18

Rahoui F., Lee J. C., Heinz S., Hines D. C., Pottschmidt K., Wilms J., Grinberg V., 2011, ApJ, 736, 63

Reid M. J., McClintock J. E., Narayan R., Gou L., Remillard R. A., Orosz J. A., 2011, ApJ, 742, 83

Rieger F. M., Bosch-Ramon V., Duffy P., 2007, in Paredes J. M., Reimer O., Torres D. F., eds, The Multi-Messenger Approach to High-Energy Gamma-Ray Sources. Springer Netherlands, Dordrecht, p. 119

Rieger F. M., Duffy P., 2004, ApJ, 617, 155

Rodriguez J. et al., 2015, ApJ, 807, 17

Romero, Vieyro F. L., Chaty S., 2014, A\&A, 562, L7

Romero G. E., Boettcher M., Markoff S., Tavecchio F., 2017, Space Sci. Rev., 207,5

Rushton A. et al., 2012, MNRAS, 419, 3194

Rushton A. P. et al., 2017, MNRAS, 468, 2788

Russell T. D. et al., 2019, ApJ, 883, 198

Rybicki G. B., Lightman A. P., 2008, Radiative Processes in Astrophysics. John Wiley \& Sons, New York

Sell P. H. et al., 2014, MNRAS, 446, 3579

Sironi L., Petropoulou M., Giannios D., 2015, MNRAS, 450, 183

Sironi L., Spitkovsky A., 2009, ApJ, 698, 1523

Sironi L., Spitkovsky A., 2014, ApJ, 783, L21

Spruit H. C., Daigne F., Drenkhahn G., 2001, A\&A, 369, 694

Stirling A. M., Spencer R. E., de la Force C. J., Garrett M. A., Fender R. P., Ogley R. N., 2001, MNRAS, 327, 1273

Strüder L. et al., 2001, A\&A, 365, L18

Szostek A., Zdziarski A. A., 2007, MNRAS, 375, 793

Tavani M. et al., 2009, Nature, 462, 620

Tavecchio F., Maraschi L., Ghisellini G., 1998, ApJ, 509, 608

Tchekhovskoy A., Narayan R., McKinney J. C., 2011, MNRAS, 418, L79

Tetarenko A. J., Casella P., Miller-Jones J. C. A., Sivakoff G. R., Tetarenko B. E., Maccarone T. J., Gandhi P., Eikenberry S., 2019, MNRAS, 484, 2987

Tomsick J. A. et al., 2013, ApJ, 780, 78

Uttley P., 2017, in Ness J.-U., Migliari S., eds, The X-ray Universe 2017, XRU. p. 230

Virtanen P. et al., 2020, NatMe, 17, 261

Waggett P. C., Warner P. J., Baldwin J. E., 1977, MNRAS, 181, 465

Walter R., Xu M., 2017, A\&A, 603, A8

Webster B. L., Murdin P., 1972, Nature, 235, 37

Wik D. R. et al., 2014, ApJ, 792, 48

Zanin R., Fernández-Barral A., de Oña Wilhelmi E., Aharonian F., Blanch O., Bosch-Ramon V., Galindo D., 2016, A\&A, 596, A55

Zdziarski A. A., Bottcher M., 2015, MNRAS, 450, L21

Zdziarski A. A., Lubiński P., Sikora M., 2012, MNRAS, 423, 663

Zdziarski A. A., Malyshev D., Chernyakova M., Pooley G. G., 2017, MNRAS, 471,3657

Zdziarski A. A., Pjanka P., Sikora M., Stawarz Ł., 2014, MNRAS, 442, 3243

Zhang J., Xu B., Lu J., 2014, ApJ, 788, 143

Ziółkowski J., 2014, MNRAS, 440, L61

This paper has been typeset from a $\mathrm{T}_{\mathrm{E}} \mathrm{X} / \mathrm{L} \mathrm{T}_{\mathrm{E}} \mathrm{X}$ file prepared by the author. 